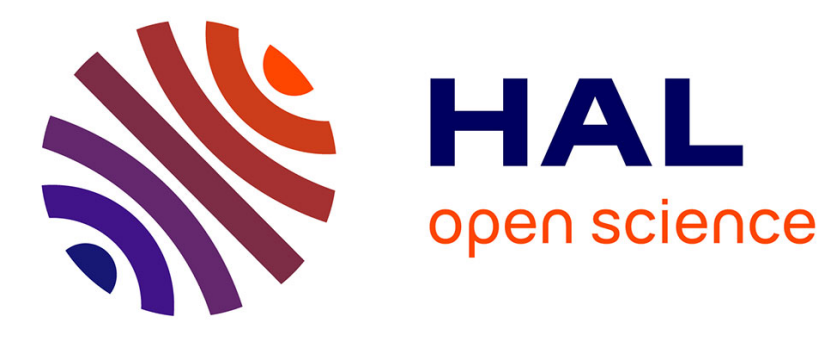

\title{
Deterministic-random separation in nonstationary regime
}

Dany Abboud, Jérôme Antoni, Sophie Sieg-Zieba, Mario Eltabach

\section{To cite this version:}

Dany Abboud, Jérôme Antoni, Sophie Sieg-Zieba, Mario Eltabach. Deterministic-random separation in nonstationary regime. Journal of Sound and Vibration, 2016, Journal of Sound and Vibration, 362, pp.305-326. 10.1016/j.jsv.2015.09.029 . hal-01285390

\section{HAL Id: hal-01285390 \\ https://hal.science/hal-01285390}

Submitted on 3 Sep 2021

HAL is a multi-disciplinary open access archive for the deposit and dissemination of scientific research documents, whether they are published or not. The documents may come from teaching and research institutions in France or abroad, or from public or private research centers.
L'archive ouverte pluridisciplinaire HAL, est destinée au dépôt et à la diffusion de documents scientifiques de niveau recherche, publiés ou non, émanant des établissements d'enseignement et de recherche français ou étrangers, des laboratoires publics ou privés. 


\title{
Deterministic-random separation in nonstationary regime
}

\author{
D. Abboud ${ }^{\mathrm{a}, \mathrm{b}, *}$, J. Antoni $^{\mathrm{a}}$, S. Sieg-Zieba ${ }^{\mathrm{b}}$, M. Eltabach ${ }^{\mathrm{b}}$ \\ a Laboratoire Vibrations Acoustique (LVA), Université de Lyon (INSA), F-69621 Villeurbanne Cedex, France \\ b Technical Center of Mechanical Industries (CETIM), CS 80067, 60304 Senlis Cedex, France
}

\section{A R T I C L E I N F O}

\section{Article history:}

Received 4 November 2014

Received in revised form

15 September 2015

Accepted 18 September 2015

Handling Editor: K. Shin

Available online 31 October 2015

\begin{abstract}
A B S T R A C T
In rotating machinery vibration analysis, the synchronous average is perhaps the most widely used technique for extracting periodic components. Periodic components are typically related to gear vibrations, misalignments, unbalances, blade rotations, reciprocating forces, etc. Their separation from other random components is essential in vibration-based diagnosis in order to discriminate useful information from masking noise. However, synchronous averaging theoretically requires the machine to operate under stationary regime (i.e. the related vibration signals are cyclostationary) and is otherwise jeopardized by the presence of amplitude and phase modulations. A first object of this paper is to investigate the nature of the nonstationarity induced by the response of a linear time-invariant system subjected to speed varying excitation. For this purpose, the concept of a cyclo-non-stationary signal is introduced, which extends the class of cyclostationary signals to speed-varying regimes. Next, a "generalized synchronous average" is designed to extract the deterministic part of a cyclo-non-stationary vibration signal-i.e. the analog of the periodic part of a cyclostationary signal. Two estimators of the GSA have been proposed. The first one returns the synchronous average of the signal at predefined discrete operating speeds. A brief statistical study of it is performed, aiming to provide the user with confidence intervals that reflect the "quality" of the estimator according to the SNR and the estimated speed. The second estimator returns a smoothed version of the former by enforcing continuity over the speed axis. It helps to reconstruct the deterministic component by tracking a specific trajectory dictated by the speed profile (assumed to be known a priori).The proposed method is validated first on synthetic signals and then on actual industrial signals. The usefulness of the approach is demonstrated on envelope-based diagnosis of bearings in variable-speed operation.
\end{abstract}

(c) 2015 Elsevier Ltd. All rights reserved.

\section{Introduction}

In most mechanical systems, gears and rolling-element bearings constitute the core elements of the power transmission chain. Therefore, their diagnosis is critical to ensure the security of the equipment and avoid possible breakdowns in the

\footnotetext{
Abbreviations: CS, cyclostationary; CS1, first-order cyclostationary; CS2, second-order cyclostationary; SA, synchronous average; LTI, linear timeinvariant; CNS, cyclo-non-stationary; DRS, deterministic random separation; GSA, generalized synchronous average; dof, degree-of-freedom; KDE, kernel density estimation; SES, squared envelope spectrum

* Corresponding author.

E-mail addresses: dany.abboud@insa-lyon.fr, dany.abboud@cetim.fr, d-abboud@live.com (D. Abboud), Jerome.antoni@insa-lyon.fr (J. Antoni), sophie.sieg-zieba@cetim.fr (S. Sieg-Zieba), mario.eltabach@cetim.fr (M. Eltabach).
} 


\section{Nomenclature}

card $\left\{K_{r}\right\}$ number of cycles belonging to regime $r$ $K_{r} \quad$ set of cycle indices belonging to regime $r$ $m_{Y}(\bar{\theta}) \quad$ SA of $Y$

$m_{Y}(\bar{\theta}, \omega)$ GSA of $Y$

$m_{Y}(\theta) \quad$ GSA trajectory tracked for a given speed profile $\omega=\omega(\theta)$

$\widehat{m}_{Y}\left(\bar{\theta}, \omega_{r}\right)$ raw estimator of the GSA of $Y$ at $\omega_{r}$

$\widehat{m}_{Y}(\bar{\theta}, \omega)$ smoothed estimator of the GSA of $Y$

$P_{Y}\left(\bar{\theta}, \omega_{r}\right)$ mean instantaneous power of $Y$ at $\omega_{r}$ $t$

$\delta \omega$

$\Delta \omega$

$\mathbb{E}\{*\}$

$\mathbb{E}\{* \mid A\}$

$\theta$

$\bar{\theta}$

$\Theta$

$\omega_{r}$ time variable

speed resolution

speed varying margin

ensemble averaging operator

ensemble average operator conditioned to

event $A$

angle variable

angular location in the cycle: $\bar{\theta} \epsilon[0, \Theta]$

angular period

central angular frequency at regime $r$

system. Interestingly, it was shown that these components-when operating under constant regime-produce symptomatic cyclostationary (CS) vibrations that can be identified and characterized by dedicated tools [1]. Specifically, gears are likely to generate first-order cyclostationary (CS1) vibrations characterized by a periodic mean, whereas rolling-element bearings generate second-order cyclostationary (CS2) vibrations characterized by a periodic autocovariance function. The former are deterministic in nature, whilst the latter are random. Their separation is of high practical importance for differential diagnosis [2-4].

In this context, the synchronous average (SA) is a powerful tool for extracting periodic components [5,6]. Based on prior knowledge of the desired component, it consists of segmenting the temporal signal into blocks of length equal to the signal period and averaging them together to extract the periodic waveform. The "residual signal"-which constitutes the random part-is then obtained by subtracting the estimated periodic part from the original signal. The principle of the SA assumes the periodic waveform to be stable in time, which in turn requires a constant speed. However, in practice, such a condition is difficult to obtain: the operating speed often undergoes some fluctuations which are likely to jeopardize the effectiveness of the SA, even if very low. Since repetitive patterns in rotating machines are intrinsically locked to specific angular positions, it thus makes sense to synchronize the SA with respect to angle rather than to time. This problem has found several practical solutions such as angular sampling or resampling (also known as "computed order tracking" [7]): whereas the former directly acquires the signal at constant angular intervals, the latter uses a tachometer to resample the time signal at constant angular increments by interpolation [8,9]. In this case, the cyclostationary property holds in the angle domain and, consequently, the SA is applied on the (resampled) signal. Incidentally, this facilitates the averaging operation since each angular period then comprises the same (whole) number of angular increments. Nevertheless, the change from a temporal to an angular variable raises issues about the actual nature of cyclostationarity of rotating machine signals. This was for instance addressed in Ref. [10] where the authors explored the concept of angle-CS signals and provided the conditions of its equivalence with the time-CS.

In the case of high speed fluctuations, signals are subjected to significant distortions that jeopardize the effectiveness of the SA. These distortions are basically introduced by (i) variations of the machine power intake and (ii) the effect of linear time-invariant (LTI) transfers. Whereas the first effect essentially results in amplitude modulation, the second one also induces phase modulation. The latter phenomenon was originally inspected in Ref. [11], before being revisited in Ref. [12] through a comprehensive theoretical analysis. Non-periodic modulations obviously invalidate the (angle-) CS assumption and call for a more general description of nonstationary signals. In accordance, "cyclo-non-stationarity" (CNS), a new class recently introduced in Ref. [13], appears to be a good candidate to describe speed-varying signals: it enfolds highly nonstationary signals undergoing long-term structural changes in their properties, yet preserving at the same time short-term cyclic rhythms locked to the angular evolution.

The consideration of CNS signals requires the revision of existing CS tools and in particular of the SA. In order to accommodate the SA with the phase blur resulting from the transmission path effect, Stander and Heyns [11] proposed an enhanced version coined "phase domain averaging"-comprising a phase correction of the cycles before the averaging operation. The phase information was returned by Hilbert demodulation of a high-energy harmonic (such as a meshing order) of the resampled acceleration signal. Later, a simpler variant was provided in Ref. [14], coined the "improved synchronous average", which consists in resampling the signal with a virtual tachometer signal synthesized via the demodulated phase. This work was inspired from Ref. [9] which introduced a technique to perform angular resampling using the acceleration signal of a gearbox operating under limited speed fluctuation.

Recently, the same technique was used in Ref. [12] to identify the optimal demodulation band for deterministic/random separation (DRS) in speed varying conditions. Moreover, the authors in Ref. [15] provided a parametric approach in an attempt to generalize the SA to the CNS case. Using Hilbert space representation, they decomposed the deterministic components on to a set of periodic functions multiplied by speed-dependent functions apt to capture long-term evolution over consecutive cycles. Yet, their method carries the general disadvantages of parametric approaches, namely, the critical dependence on the basis order. Another attempt was made recently in Ref. [13] where the aim was to remove the deterministic part of the vibrations produced by an internal combustion engine in runup regimes. Assuming a first-order Markov 
dependence of the CNS signal, the authors introduced an operator-coined the "cyclic difference"-based on subtracting each cycle from the previous one in the resampled signal. Despite the good compliance of this method for the particular addressed application, it suffers from high variability in the more general setting.

The principal object of this paper is to provide a novel operator, coined the "generalized synchronous average" (GSA), which happens to be an extension of the classical SA to the CNS case, with the aim of enhancing DRS in variable regime. It is assumed that the rotational speed is precisely known; its estimation is beyond the scope of this paper and may be found in references such as [16-25]. This paper is organized as follows: Section 2 states the problem with a particular attention to formulating the transmission path effect from a CNS view. Section 3 introduces the GSA operator, two dedicated estimators of it and a statistical study in order to evaluate the estimator quality according to the SNR and the estimated speed. Section 4 validates the effectiveness of the GSA on numerical signals and Section 5 on actual industrial signals. Eventually, in light of the obtained results, the paper is sealed with a general conclusion in Section 6.

\section{Problem statement}

This section starts with a brief review of the SA. Then, the transmission path effect is qualitatively addressed with a link made with CNS.

\subsection{Synchronous average: a brief review}

The synchronous average (SA)-widely termed as the time synchronous average [26]-is an efficient technique for the extraction of periodic waveforms from a noisy signal. It consists of averaging periodic sections of the signal-known as cycles -assuming a priori knowledge of the period. Under the assumption of cycloergodicity [10], the SA is perhaps the best candidate for estimating the mean function of a CS signals. In practice, it is however jeopardized when applied in the timedomain due to existing speed fluctuations or if the signal period is not a multiple of the sampling period. In such cases, angular resampling can be a simple preprocessing solution consisting of expressing the signal in terms of the angular variable $\theta$ of the machine instead of time $t$. Precisely, let $Y(\theta)$ be an angle-CS signal of cycle $\Theta$ (for instance $\Theta=2 \pi$ ); its SA is then defined as

$$
m_{Y}(\bar{\theta})=\lim _{M \rightarrow \infty} \frac{1}{2 M+1} \sum_{m=-M}^{M} Y(\bar{\theta}+m \Theta)
$$

where $(2 M+1)$ stands for the number of averaged cycles and $\bar{\theta} \in[0, \Theta]$ for the angular location in the cycle (i.e. $\bar{\theta}=\theta-k \Theta$ with $k$ the greatest integer smaller than $\theta / \Theta$ ). The SA is widely used in vibration analysis of rotating machinery to separate deterministic waveforms (such as produced by imbalances, misalignments, anisotropic rotors, flexible coupling, gear meshing and other phenomena) from other competing but random sources.

\subsection{Transmission path effect}

A primary goal in machine diagnostics is to infer the cause of abnormal vibrations. In most cases, accelerometers are remoted from the sources due to non-intrusion constraints and there exists a transmission path effect characterized by the transfer function of the structure. In cases where the sensor is intentionally used outside its nominal bandwidth (e.g. as a shock sensor), its transfer function should be considered as well. Consequently, the measured signal is to be interpreted as the output of an LTI system. This system is characterized by ordinary differential equations in time, independently of the excitation nature. As a result, the response of the system to a complex exponential temporal waveform is kept unchanged except for a phase shift and an amplitude amplification characterized by the system transfer function. In this case, the transmission path induces maximum amplification and phase shift when the frequency content of the excitation coincides with a resonance of the transfer function. This also holds true in general for time-CS signals: a LTI system excited by a timeCS signal returns a time-CS output. However, the situation is not the same for angle-CS signals. In the case of large speed variations, the corresponding response is instantaneously delayed and amplified/attenuated [11] according to the fluctuating amplitude and frequency content of the input signal. From a temporal view, the system response is the convolution of a nonstationary excitation with a LTI transfer function: it is therefore nonstationary as well. Conversely, from an angular point of view the order content of the excitation remains constant, yet the transfer function becomes angle-varying; therefore, the response of the system to an angle-CS excitation is CNS in general, and angle-CS in the particular case of periodic, stationary or cyclostationary operating speed [10] (see Fig. 1).

The present paper is mainly concerned with angle-CS1 excitation and the related transmission path effect on gear vibrations which jeopardizes the (traditional) SA. The issue can be explained from different but equivalent points of view. First, according to the previous discussion, the system response is no longer angle-CS1, which invalidates the working assumption of the SA [12]. Alternatively, from an angular point of view, the averaging process over multiple shaft rotations usually results in an energy loss principally caused by the induced phase blur. Finally, from the order domain point of view, 


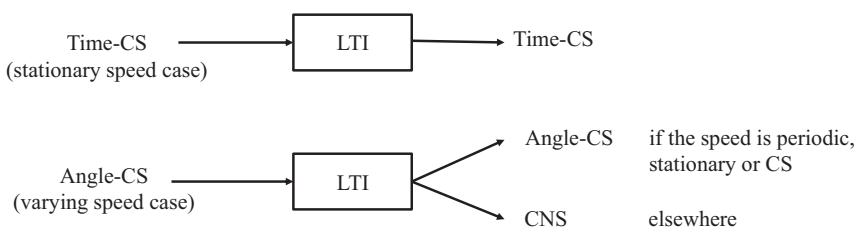

Fig. 1. Transmission path effect on cyclostationary excitations.

the SA is equivalent to applying a narrow-band comb filter [27]; yet, the amplitude distortion and the phase blur results in energy leakage outside the lobes of the comb filter.

In summary, when rotating machines undergo large speed variations, the resulting vibration signals lose their angle-CS properties; thus, invalidates the application of traditional CS tools such as the SA. The aim of the next section is to characterize such signals which preserve repetitive patterns related to the cyclic behavior of the excitation, yet with angle (or time) varying properties.

\subsection{Speed dependence}

This section introduces the basic model that will serve to define the GSA, which consists of Fourier series whose complex exponentials are function of the angle variable, $\theta$, and the coefficients are only dependent on the speed, $\omega$.

To avoid confusions between angle and time domains, the accent mark "tilde" will refer to the temporal representation of the signal. Building on the discussion of Section 2.2, a CNS process can be seen as the response $\tilde{Y}(t)=Y(t(\theta))$ of a LTI system, say $\tilde{h}(t)$, to an angle-CS excitation

$$
\tilde{X}(t)=X(\theta(t))=\sum_{\alpha} c_{X}^{\alpha}(\theta(t)) \mathrm{e}^{\mathrm{j} 2 \pi \alpha \frac{\theta(t)}{\theta}}
$$

where $\Theta$ stands for the angular period of $X(\theta(t)), c_{X}^{\alpha}(\theta)$ are mutually (angle-) stationary Fourier coefficients and $\theta(t)=\int_{0}^{t} \omega(t) \mathrm{d} t$ is the angular position of the reference. In short

$$
\tilde{Y}(t)=\tilde{h}(t) \otimes \tilde{X}(t),
$$

where $\otimes$ stands for the convolution product. In the particular case of constant operating speed (i.e. $\left.\theta(t)=\omega_{0} t\right)$ the response takes the particular form

$$
Y(\theta)=\sum_{\alpha} c_{Y}^{\alpha}\left(\theta, \omega_{0}\right) \mathrm{e}^{\mathrm{j} 2 \pi \alpha \frac{\theta(t)}{\theta}}=\sum_{\alpha}\left(c_{X}^{\alpha}(\theta) A\left(\alpha \omega_{0} / 2\right) e^{\mathrm{j} k \Phi\left(\alpha \omega_{0} / 2\right)}\right) \mathrm{e}^{\mathrm{j} 2 \pi \alpha \frac{\theta}{\theta}}, \quad \alpha \in \mathbb{Z},
$$

where $A(f) \mathrm{e}^{\mathrm{j} k \Phi(f)}=H(f)$ is the system frequency response function. Actually, Eq. (4) reflects the pivotal role of the speed not only on the amplitude, but also on the phase of the system response.

In the case of speed varying excitation, the response would turn into a convolution between an angle-CS excitation and an LTI system, which is less straightforward than Eq. (4) because the input signal would no longer be time-CS in general. One alternative would be to express the convolution directly in the angular domain, i.e. symbolically

$$
Y(\theta)=h(\theta) \otimes Y(\theta),
$$

where $h(\theta)=\tilde{h}(t(\theta))$ would then become angle-varying owing to the nonlinearity of the angle-time relationship. In this case, the Fourier coefficients of the response at a given instant are principally dependent on the operating speed at that instant, as well as past and future instances of the speed profile. This implies that, beside the operating speed, the Fourier coefficients of the response are also dependent on its higher derivatives at the evaluated instant, i.e.

$$
\tilde{Y}(t)=\sum_{\alpha} c_{Y}^{\alpha}(\theta(t), \omega(t), \dot{\omega}(t), \ddot{\omega}(t), \dddot{\omega}(t) \ldots) \mathrm{e}^{\mathrm{j} 2 \pi \alpha^{\theta(t)}} .
$$

Under mild conditions, the dependence on higher derivative orders can be neglected and the variations of both amplitude and phase are consequently modeled with an explicit dependence on the speed only:

$$
\tilde{Y}(t)=\sum_{\alpha} c_{Y}^{\alpha}(\theta(t), \omega(t)) \mathrm{e}^{\mathrm{j} 2 \pi \alpha \frac{\theta(t)}{\theta}},
$$

where $c_{Y}^{\alpha}(\theta, \omega)$ stands for the complex Fourier coefficients having a speed-dependent joint probability density function-i.e. mutually stationary stochastic process for a constant speed $\omega$. The angular representation of a CNS signal can be deduced as

$$
Y(\theta)=\sum_{\alpha} c_{Y}^{\alpha}(\theta, \tilde{\omega}(\theta)) \mathrm{e}^{\mathrm{j} 2 \pi \alpha \frac{\theta}{\theta}}
$$

with $\tilde{\omega}(\theta)=\omega(t(\theta))$. Note that Eq. (7) is perfectly valid for runup regimes (where higher-order derivatives of the speed are nil) and approximately valid in the case of modest accelerations (i.e. smooth variability of the speed profile). This model accounts for changes in the signal structure by introducing a dependency of the Fourier coefficient on speed, while the cyclic 
behavior expressed by the complex exponential basis is kept invariant. Therefore, the deterministic part of the system response-which will be lately extracted by the GSA-shows an explicit dependence on the speed and is expressed in the angular domain as

$$
M_{1 Y}(\theta)=\mathbb{E}\{Y(\theta)\}=\sum_{\alpha} \bar{c}_{Y}^{\alpha}(\omega(\theta)) \mathrm{e}^{\mathrm{j} 2 \pi \alpha \frac{\theta}{\theta}}
$$

where $M_{1 Y}(\theta)$ denotes the first-order moment of $Y(\theta)$, and

$$
\bar{c}_{Y}^{\alpha}(\omega(\theta))=\mathbb{E}\left\{c_{Y}^{\alpha}(\theta, \omega) \mid \omega=(\theta)\right\}
$$

denotes the expected value of $c_{Y}^{\alpha}(\theta, \omega)$ conditioned on $\omega(\mathbb{E}\{* \mid a\}$ stands for the expected value of $*$ conditioned to $a)$. The importance of this model is to characterize the nonstationarity of the response and to highlight the effect of the speed on the signal structure. Hence, the consideration of the speed seems to be compulsory for the investigation of the statistical moments and, particularly, the mean value. The aim of the next section is to link this model with the CNS class.

\subsection{Link with cyclo-non-stationarity}

Cyclo-non-stationarity has been recently introduced [13] in an attempt to generalize the CS class to account for possible long-term variations in the signal statistics while maintaining short-term cyclic rhythms. The model of Eq. (7) is accordance with such a definition. Specifically, let $\Theta$ be the angular period of the excitation; then, building on Eq. (7), the probability density function of $Y(\theta)$,

$$
f_{Y}(y)=f_{Y}(y ; \bar{\theta}, \omega)
$$

is dependent on both $\bar{\theta}$ and $\omega(\bar{\theta}$ is the angular position in the cycle defined in Section 2.1). In particular, the conditional first-order moment reads

$$
M_{1 Y}(\bar{\theta}, \omega)=\mathbb{E}\{Y(\theta) \mid \theta / \Theta=\bar{\theta}, \omega(\theta)=\omega\}=\sum_{\alpha} \bar{c}_{Y}^{\alpha}(\omega) \mathrm{e}^{\mathrm{j} 2 \pi \alpha \frac{\bar{\theta}}{\bar{\theta}},}
$$

where $[\theta / \Theta]$ stands for the remainder of the fraction $\theta / \Theta$, which evidences a dependence of the Fourier coefficients on speed; this defines a CNS process [28]. ${ }^{1}$

\section{The proposed solution}

Cyclo-non-stationarity brings to the fore an essential difference between ensemble averaging and angle averaging. The former means that averaging is applied over all realizations of a stochastic process, whereas the latter implies that averaging is applied over the time (or angle) variable of a particular realization. In fact, their equivalence is of high practical concern in most physical applications wherein only one realization is usually available. For CS signals, this is guaranteed by the cycloergodic assumption under which the ensemble average can be replaced by the SA. The primary aim of the present section is to introduce an angular operator equivalent to the ensemble average in the CNS case. A consistent estimator is also provided and a brief statistical study is then performed to assess its performance.

\subsection{Generalized synchronous average}

As previously shown, the statistics of a CNS signal instantaneously change with speed and thus lose their periodicity. As a consequence, the validity of cyclic averaging is jeopardized. For this reason, angle averaging should be redefined to account for the speed dependence and accommodate for the induced changes in the signal. To do so and according to Eq. (11), the idea is to perform the cyclic average on samples pertaining to the same speed. Thus, one can extent the SA to CNS signals by introducing a more general operator coined the generalized synchronous average (GSA). Given a CNS signal $Y(\theta)$ with angular period $\Theta$ and operating under the speed profile $\omega(\theta)$, the GSA is defined as

$$
m_{Y}(\bar{\theta}, \omega)=\lim _{\delta \omega \rightarrow 0} \lim _{\operatorname{card}\left\{K_{\bar{\theta}, \omega}\right\} \rightarrow \infty} \frac{1}{\operatorname{card}\left\{K_{\bar{\theta}, \omega}\right\}} \sum_{k \in K_{\bar{\theta}, \omega}} Y(\bar{\theta}+k \Theta),
$$

where $\delta \omega$ stands for the speed resolution and $K_{\bar{\theta}, \omega}=\left\{k \in \mathbb{N} \mid \omega-\frac{\delta \omega}{2} \leq \omega(\bar{\theta}+k \Theta)<\omega-\frac{\delta \omega}{2}\right\}$ stands for the set of samples located at $\bar{\theta}$ and coinciding with speed values in the interval $\left[\omega-\frac{\delta \omega}{2} ; \omega-\frac{\delta \omega}{2}\right]$. This operator extracts the first-order CNS (CNS1) "field" corresponding to the deterministic components of a signal for all the existing operating regimes by explicitly

\footnotetext{
${ }^{1}$ In this reference, a CNS process is defined by a Fourier decomposition of nonstationary complex Fourier coefficient. It is worth noting that this definition is rather symbolic since it actually comprises all nonstationary signals, the reason it was not adopted by the authors in the present paper.
} 


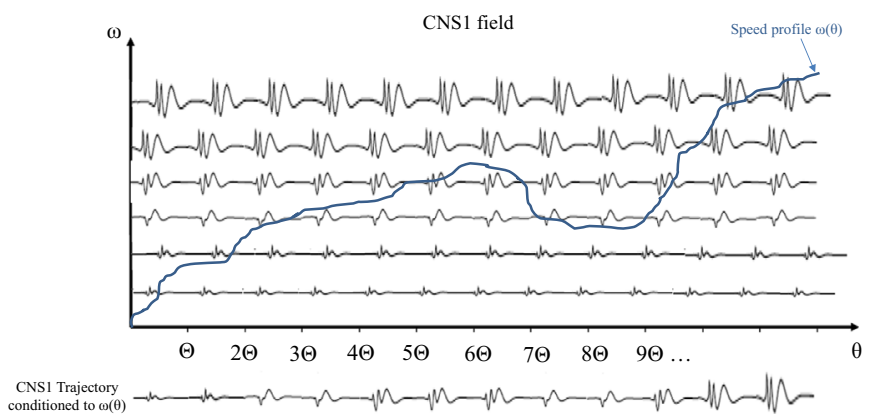

Fig. 2. Trajectory of a CNS1 signal conditioned to a given speed profile $\omega(\theta)$. Each horizontal slice in this field associated with a fixed speed value $\omega$ represents a CS process with specific statistics. A CNS process is then a trajectory in this field defined by the speed profile.

accounting for the speed variable. Note that, in the case of constant speed, say $\omega_{0}$, the set $K_{\bar{\theta}, \omega_{0}}$ comprises all the cycles for all $\bar{\theta}$ and the GSA boils down to the classical SA.

Note that the GSA is in general a function of two variables, i.e. angle and speed. Given a particular speed profile $\omega(\theta)$, the "trajectory" of the CNS1 component of $Y(\theta)$ is thus returned by

$$
m_{Y}(\theta)=m_{Y}(\bar{\theta}=\theta / \Theta, \omega=\omega(\theta))
$$

viewed as a function of $\theta$ only-see Fig. 2. Having introduced the GSA, it now remains to propose a consistent estimator that applies to finite-length signals. This is addressed in the next section.

\subsection{Estimation issues}

\subsubsection{Raw estimator}

The practical implementation of the GSA faces several difficulties. In particular, the finite length of the signal prevents the speed resolution to be infinitely narrow and the number of averages to be infinitely large. Furthermore, the cyclic averaging operation is pointwise, which makes difficult its application in practice. A natural way to overcome this limitation is to assume a stationary behavior of the machine regime in each interval $[\omega-\delta \omega / 2 ; \omega+\delta \omega / 2]$ for a given $\delta \omega>0$ (i.e. local stationarity of the speed profile). Stated differently, the signal is supposed to be locally cyclostationary at those speeds. In details, the speed profile is divided into a predefined set of speed intervals called regimes defined by their central frequency $\omega_{r}$ and the speed resolution $\delta \omega$. Then, each cycle is associated to the regime that is closest to its mean speed value. The raw estimator of the GSA of a finite-length CNS signal $Y(\theta)$, with angular period $\Theta$ and operating speed profile $\omega(\theta)$, is then defined as

$$
\widehat{m}_{Y}\left(\bar{\theta}, \omega_{r}\right)=\frac{1}{\operatorname{card}\left\{K_{r}\right\}} \sum_{k \in K_{r}} Y(\bar{\theta}+k \Theta),
$$

where $K_{r}=\left\{k \in \mathbb{N}^{*} \mid \omega_{r}-\frac{\delta \omega}{2} \leq \frac{1}{\Theta} \int_{(k-1) \Theta}^{k \Theta} \omega(\theta) \mathrm{d} \theta<\omega_{r}-\frac{\delta \omega}{2}\right\}$ stands for the set of cycles whose mean speed falls in the speed interval centered at $\omega_{r}$.

When applied on vibration signals, Eq. (15) gives partial but important information about the vibration response at speed $\omega_{r}: \widehat{m}_{Y}\left(\bar{\theta}, \omega_{r}\right)$ is the estimated deterministic response at $\omega_{r}$, as if the machine was operating steadily at the corresponding central frequency. The flow-chart of the raw GSA estimator is illustrated in Fig. 3.

Note that the raw estimate of the GSA provides only estimates at the central frequencies of the predefined speed intervals. The next section provides a solution to recover the CNS1 component at any speed value to solve this issue.

\subsubsection{Smoothed estimator}

In order to overcome the discrete nature of the raw estimator, an appropriate interpolation method is used to enforce the continuity along the speed axis by means of few GSA evaluations. In this context, the kernel density estimation (KDE) method-also termed the Parzen-Rosenblatt window method-provides a non-parametric solution for an efficient smoothing operation $[29,30]$. In particular, the smoothed estimator can be obtained for all speeds from Eq. (15) by applying the KDE

$$
\widehat{m}_{Y}(\bar{\theta}, \omega)=\frac{1}{\sum_{r=1}^{R} \operatorname{kern}\left(\frac{\omega-\omega_{r}}{\lambda}\right)} \sum_{r=1}^{R} \operatorname{kern}\left(\frac{\omega-\omega_{r}}{\lambda}\right) \widehat{m}_{Y}\left(\bar{\theta}, \omega_{r}\right)
$$

where $\operatorname{kern}(*)$ is the kernel and $\lambda$ is a smoothing parameter called the bandwidth. Note that the denominator is added to ensure energy conservation. In this context, there are a variety of commonly used kernels such as uniform, triangular, normal, and others. The normal kernel is the most widely used due its good performances and mathematical convenience. 


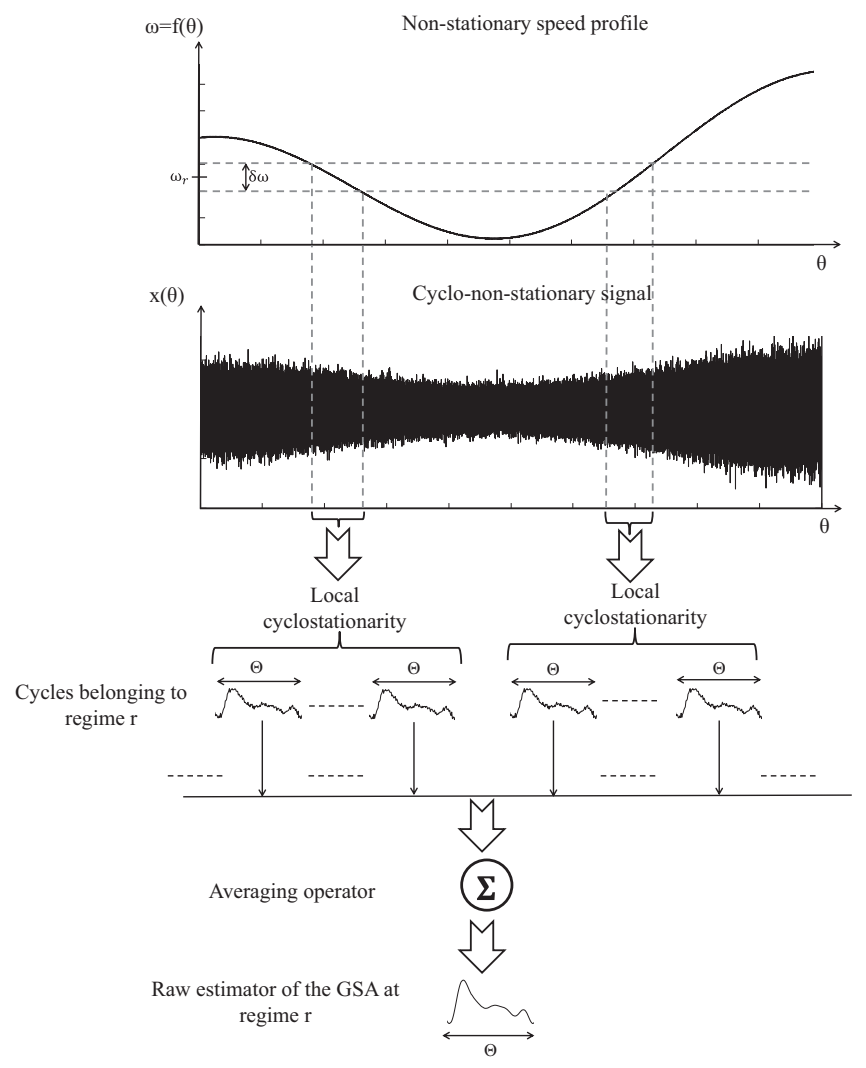

Fig. 3. Flow-chart of the GSA raw estimator.

Now having a continuous distribution along the speed axis, the estimator of the deterministic trajectory of the signal can be deduced from the smoothed estimator

$$
\widehat{m}_{Y}(\theta)=\widehat{m}_{Y}(\bar{\theta}=\theta / \Theta, \omega=\omega(\theta)) .
$$

or directly from the smoothed raw estimator

$$
\widehat{m}_{Y}(\theta)=\frac{1}{\sum_{r=1}^{R} \operatorname{kern}\left(\frac{\omega(\theta)-\omega_{r}}{\lambda}\right)} \sum_{r=1}^{R} \operatorname{kern}\left(\frac{\omega(\theta)-\omega_{r}}{\lambda}\right) \widehat{m}_{Y}\left(\bar{\theta}, \omega_{r}\right)
$$

where $\widehat{m}_{Y}\left(\theta, \omega_{r}\right)$ is the periodized version of $\widehat{m}_{Y}\left(\bar{\theta}, \omega_{r}\right)$ over the actual number of periods of $Y(\theta)$. It is worth noting that Eq. (18) requires less computational efforts than Eq. (17), though being actually equivalent (indeed, the latter requires the assessment of all $(\bar{\theta}, \omega)$ couples, whereas the former tracks a specific trajectory in the $(\bar{\theta}, \omega)$ plane). Interestingly, the estimator of Eq. (17) is reminiscent to the "functional" form originally proposed in Ref. [15]; yet it presents numerous advantages as compared to the latter: in particular, it does neither require to set a model order nor to solve a large system of equations. The flow-chart of this method is provided in Fig. 4.

\subsection{Statistical performances}

Since all signals are finite-length in practice, the asymptotic conditions in the GSA definition (see Eq. (15) cannot be met, resulting in a bias and variance of the raw estimator. The estimation of the GSA is a similar issue as the estimation of densities (e.g. histograms) frequently encountered in the fields of statistics and data analysis. The estimation accuracy is thus directly dependent on the discretization of the data, a topic on which a last literature exists [31,32]. The aim of this section is to provide a statistical study to evaluate the estimator quality according to the SNR and the estimated speed. For this purpose, the related bias and variance will be subsequently calculated: while the former reflects its asymptotic deviation from the actual value, the latter evaluates its consistency. Since the efficiency of the smoothed estimator is conditioned by that of the raw estimator, only the latter will be assessed. Interested readers can find the proofs in Appendix. 


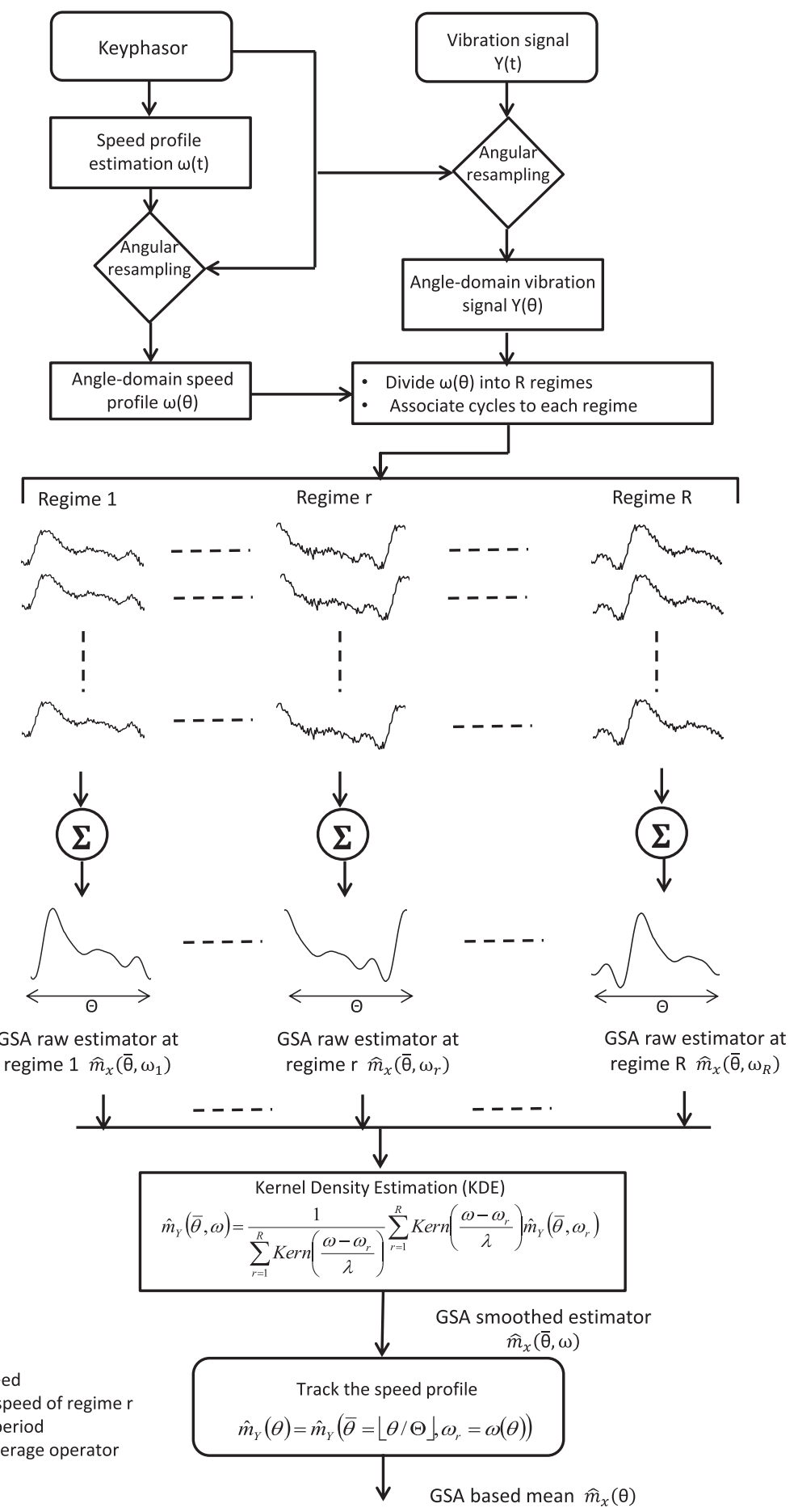

Fig. 4. Flow-chart of the proposed method.

\subsubsection{Bias}

The bias of an estimator is defined by the difference between its statistical expectation and its actual value. For the raw GSA estimator, there are basically two sources of bias stemming from (i) finite sample effects (i.e. limited card $\left\{K_{r}\right\}$ ) and (ii) the distortion caused by discretizing the speed profile. In this section, only the latter is evaluated due to its direct relation to the paper topic. Starting from the random response model provided in Eq. (7), it can be shown that the bias takes the 
particular form

$$
\operatorname{Bias}\left\{\widehat{m}_{Y}\left(\bar{\theta}, \omega_{r}\right)\right\} \approx \frac{\delta \omega^{2}}{24} m_{Y}^{\prime \prime}\left(\bar{\theta}, \omega_{r}\right)
$$

where $m_{Y}(\bar{\theta}, \omega)$ is the actual GSA of $Y(\theta)$ and $m_{Y}^{\prime \prime}(\bar{\theta}, \omega)={ }^{2}\left(m_{Y}(\bar{\theta}, \omega)\right) / \omega^{2}$. Obviously, there are two parameters that govern the bias, namely the speed resolution, $\delta \omega$, and the second derivative of the actual GSA with respect to speed. The former-which is controllable-is the most intuitive: the narrower $\delta \omega$ is, the more similar the corresponding cycle statistics are, and consequently, the more accurate the estimation is. The latter, however, is an uncontrollable parameter which requires careful interpretation. When $m_{Y}(\bar{\theta}, \omega)$ is seen as a function of $\omega$, it is systematically underestimated at peaks where $m_{Y}^{\prime \prime}\left(\bar{\theta}, \omega_{r}\right)<0$ (e.g. at resonances) and overestimated at deeps where $m_{Y}^{\prime \prime}\left(\bar{\theta}, \omega_{r}\right)>0$. In short, the estimator is asymptotically unbiased as the speed resolution is made infinitely narrow. In practice, for a finite signal length, the speed resolution should be carefully selected so that the number of averaged cycles in a regime remains sufficient.

\subsubsection{Variance}

It is of prime importance to calculate the variance of the proposed estimator in order to assess its consistency as well as the influential parameters. It can be shown that, neglecting the finite sample effect, the variance of the raw GSA estimator is

$$
\operatorname{Var}\left\{\widehat{m}_{Y}\left(\bar{\theta}, \omega_{r}\right)\right\} \approx \frac{1}{\operatorname{card}\left\{K_{r}\right\}}\left[P_{Y}\left(\bar{\theta}, \omega_{r}\right)+\frac{\delta \omega^{2}}{24} P_{Y}^{\prime \prime}\left(\bar{\theta}, \omega_{r}\right)\right],
$$

where

$$
P_{Y}(\bar{\theta}, \omega)=m_{Y_{c}^{2}}(\bar{\theta}, \omega)
$$

stands for the mean instantaneous power of the signal with $Y_{c}(\theta)=Y(\theta)-m_{Y}(\theta)$ and $P_{Y}^{\prime \prime}(\bar{\theta}, \omega)={ }^{2}\left(P_{Y}(\bar{\theta}, \omega)\right) / \omega^{2}$. Clearly, the raw GSA estimator is asymptotically consistent due to its nil variance for an infinite signal length. Also, it depends on the signal random part as well as its second derivative with respect to $\omega$ when evaluated at speed $\omega_{r}$. Moreover, the speed resolution $\delta \omega$ plays a remarkable role which can, when it is narrow enough, lessens the effect of the second derivate term. In general, the raw GSA estimator shows a typical bias-variance trade-off: to reduce the bias $\delta \omega$ must be small, whereas to reduce the variance $\delta \omega \sim 1 / \operatorname{card}\left\{K_{r}\right\}$ must be high. Thus, the only way to reduce both the bias and the variance is by increasing the signal length.

Eventually, an obvious assumption in this estimation is that the accuracy of the available rotation speed is superior to the speed resolution $\omega$ used for the discretizing the speed in the GSA estimation. In practice, this is guaranteed by selecting an encoder with a sufficient resolution when measuring the rotation speed. Nevertheless, the limited accuracy of the measured (or estimated) rotation speed induces another source of error, a topic which is for instance investigated in Ref. [33]. The impact of this type of error on the GSA is just as for the SA and has been thoroughly studied in Refs. [10,34]. In situation with high speed fluctuations, the effect of this error is negligible as compared to that induced by the limited number of averaged cycles.

\section{Numerical validation}

The aim of this section is twofold. First, the transmission path effect and the limitation of the classical SA are numerically illustrated in a simple way. Second, the effectiveness of the GSA estimation is addressed together with its influential parameters.

\subsection{Limitation of the classical SA}

A natural way to model the effect of the transmission path in rotating machines is by exciting a single degree-of-freedom (dof) system by a constant-amplitude chirp. Though being physically simplistic, ${ }^{2}$ the chirp is chosen with constant amplitude to clearly reveal the amplitude distortion. Fig. 5(a) illustrates the time evolution of the reference instantaneous frequency $f_{i}(t)=2 t+10$, (b) represents the excitation chirp synchronous with the 15 th order i.e. $\tilde{X}(t)=\sin \left(2 \pi \cdot 15 \int_{0}^{t} f_{i}(s) \mathrm{d} s\right)$, and (c) and (d) represents the amplitude and the phase spectrum of the simulated system, respectively, with impulse response

$$
\tilde{h}(t)=\frac{A}{\omega_{d}} \mathrm{e}^{-\mathrm{j} \xi \omega_{n} t} \sin \left(\omega_{d} t\right) \tilde{U}(t),
$$

\footnotetext{
${ }^{2}$ In general, the excitation is subjected to an energy modulation owing to changes of the machine power intake as the speed varies. Yet, these changes can still be generally modeled as a speed dependent amplitude distortion in the response.
} 
(a)

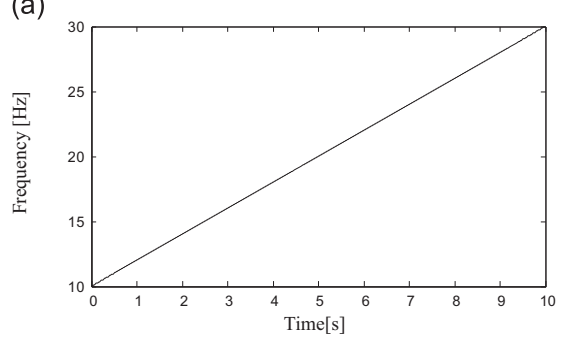

(c)

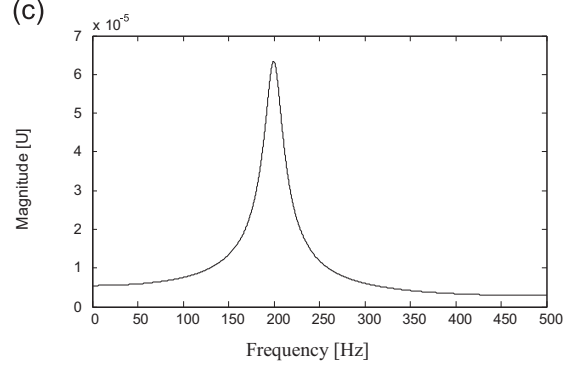

(e)
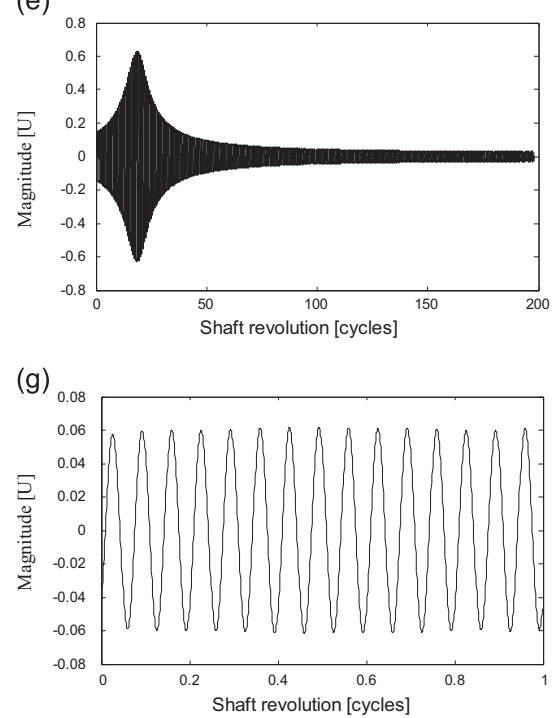

(i)

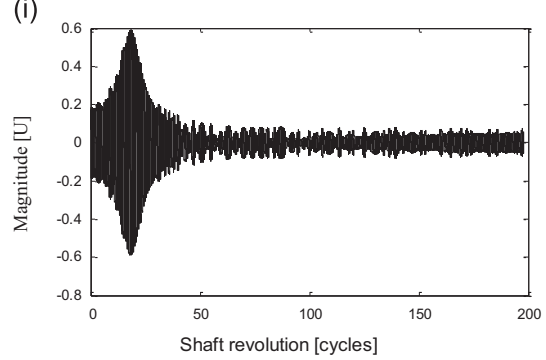

(b)

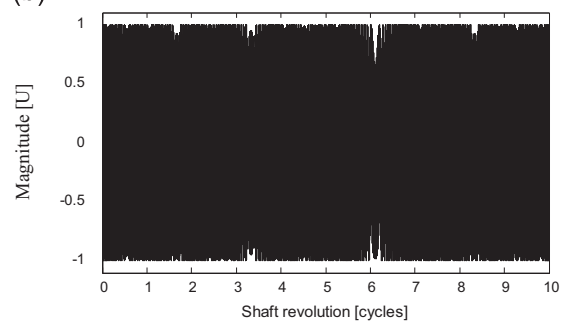

(d)

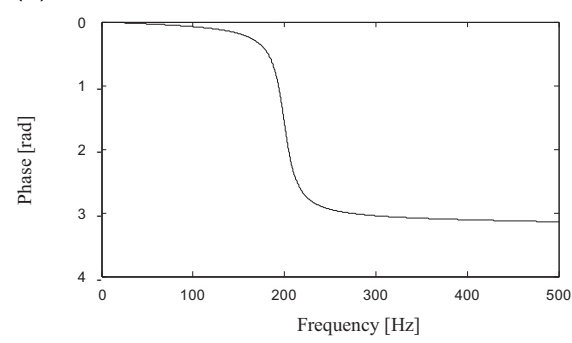

(f)
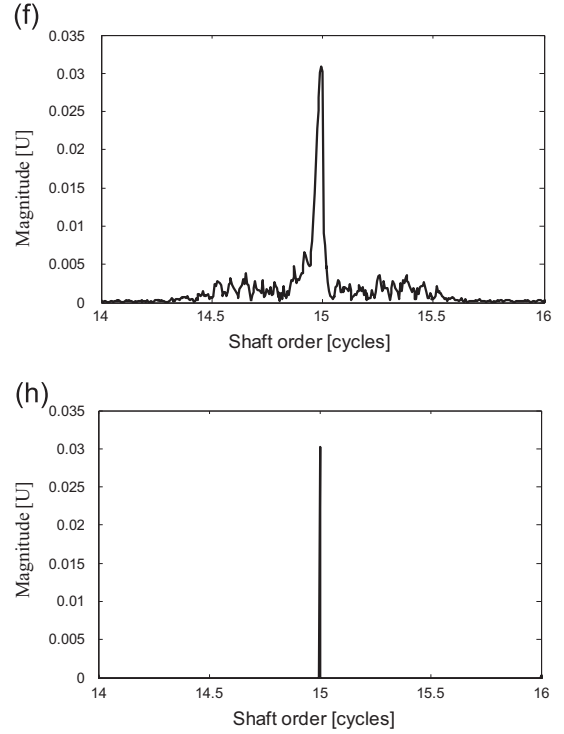

(j)

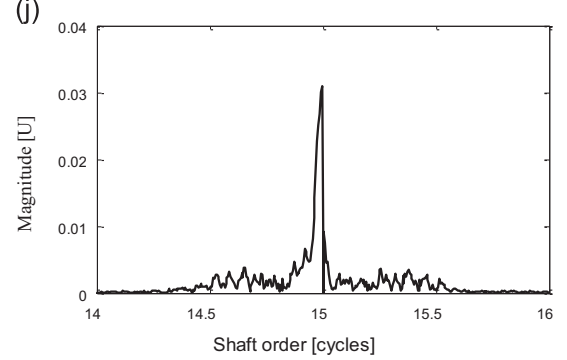

Fig. 5. Academic example illustrating the limitation of the SA when applied to a single dof system excited by a linear chirp. (a) Reference instantaneous frequency, (b) constant amplitude linear chirp synchronous with the 15th order of the reference instantaneous frequency, (c) amplitude and (d) phase spectrum of the single dof linear system, (e) angular resampled response, (f) response order spectrum, (g) SA, (h) SA order spectrum, (i) residual signal, and (j) residue order spectrum. Note that the SA energy comprises only $13.94 \%$ of the response energy.

with $A=10, \xi=0.05, \omega_{n}=2 \pi * 200, \omega_{d}=\omega_{n} \sqrt{1-\xi^{2}}$, and $\tilde{U}(t)$ the Heaviside function used to enforce the system causality. The sampling rate is set to $1 \mathrm{kHz}$ and no noise is added to evidence only the distortion effect on the SA estimation. First, a numerical convolution is performed to simulate the system response. Then, the response is resampled according to the 
reference instantaneous frequency; this is displayed in Fig. 5(e) with the corresponding order spectrum in (f). Clearly, the amplitude distortion is prominent in the angular domain and manifests itself with long-term energy modulations, whereas the phase blur is materialized by leakage in the vicinity of the excitation order 15 .

It is worth noting that the nature of the deterministic component changes at the system output (see Eq. (9): whereas the excitation is angle-periodic, the response is not albeit it conserves cyclic rhythms related to the excitation. As a matter of fact, these rhythms are captured by the complex exponential kernels in Eq. (9). Last, the SA and the corresponding residue are computed and displayed in Fig. 3(g) and (i), together with their order spectra in Fig. 5(h) and (j), respectively. Noticeably, the SA energy forms only $13.94 \%$ of the deterministic signal energy, indicating a poor estimation even in the absence of random noise.

\subsection{Evaluation of the GSA}

First, it is beneficial to illustrate the GSA on the previous simulated response. For this purpose, the speed varying margin is first divided into 20 non-overlapping regimes of $1 \mathrm{~Hz}$ width (i.e. $\delta \omega=2 \pi \mathrm{rad} / \mathrm{s}$ ) and the smoothed estimator of the GSA is applied on the noise-free signal. The smoothed parameter of the interpolation kernel (normal window) is chosen equal to the speed resolution. Results are displayed in Fig. 6 wherein (a) represents the deterministic component as well as the GSA estimate and (b) illustrates their corresponding order spectra. A significant enhancement over the SA is seen: the GSA estimate has the ability of tracking the temporal variations of the amplitude and of the phase, capturing $92.43 \%$ of the actual signal energy. It is also noticed that the estimator underestimates the actual response in the vicinity of the resonance (around cycle 20,000) as predicted by the statistical analysis of Section 3.3.1.

Second, a white Gaussian noise is added on the response to obtain a SNR $=2$ and the signal duration is enlarged 10 times (i.e. one million samples) while keeping the same speed variation. In an attempt to evaluate the influence of the speed resolution, the GSA is estimated for different interval widths ( $4 \mathrm{~Hz}, 1.5 \mathrm{~Hz}, 0.5 \mathrm{~Hz}$ and $0.33 \mathrm{~Hz}$ ). One can notice the poor estimation when performed on $4 \mathrm{~Hz}$ width, whereas significant enhancement is perceived as $\delta \omega$ decreases. With the aim of quantifying this dependence, the GSA energy is evaluated with respect to the actual response energy for different speed resolutions, $\delta \omega=\frac{\Delta \omega}{R}$ (where $\Delta \omega$ is the speed varying margin) and results are depicted in Fig. 7(b). In accordance to the statistical results obtained in Section 3.3.1, the estimated energy increases with resolution $R$ (i.e. the interval width decreases) so as to finally reach an asymptotic value. Note that the difference between the asymptotic and the ideal value (100\%) corresponds to the estimator bias for the selected parameters.

Eventually, it is noteworthy that the speed resolution cannot be taken arbitrary small because the number of averages, $\operatorname{card}\left\{K_{r}\right\}$, then decreases to zero. Therefore, a trade-off is to be made between these quantities. Accordingly, it is also beneficial to evaluate the estimation accuracy with respect to the signal length. To do so, the evolution of the estimated GSA energy is calculated with respect to the signal length for different speed resolutions, yet conserving the same speed variation (see Fig. 8). Interestingly, the obtained curves consist of a transient and a steady state: whereas the former reflects the stochastic variability of the estimator, the latter is representative of the bias. This illustrates the bias-variance trade-off.

(a)

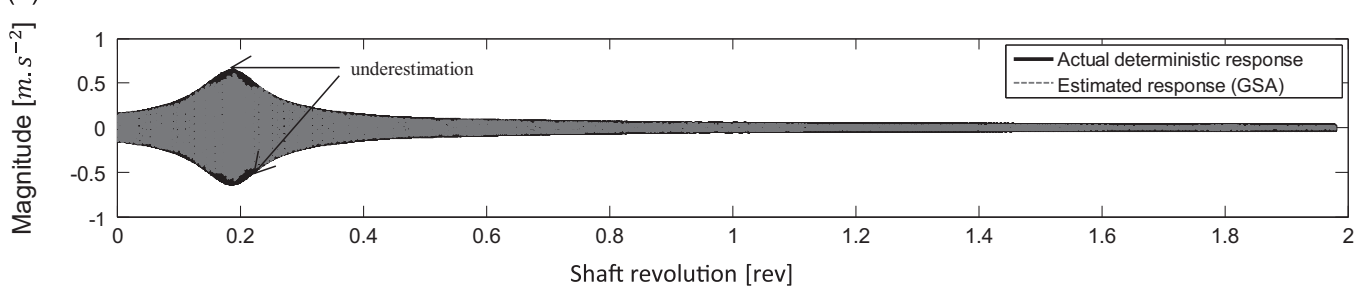

(b)

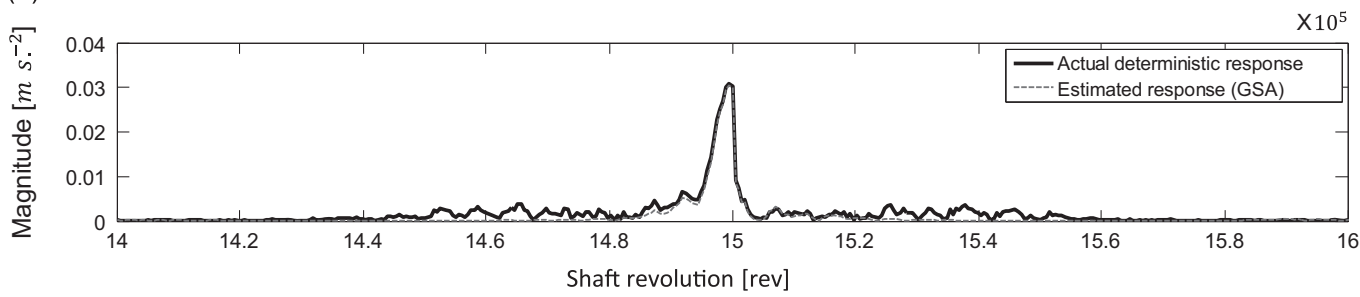

Fig. 6. (a) Actual deterministic response and GSA estimate together with their (b) order spectra. The estimation comprises $92.43 \%$ of the energy of the actual response. 
(a)

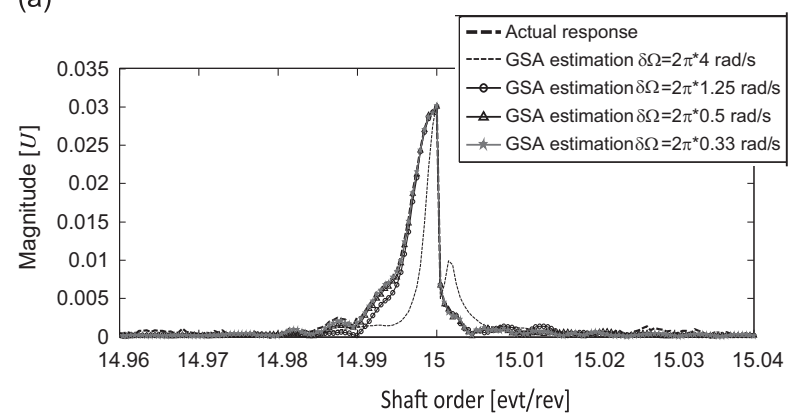

(b)

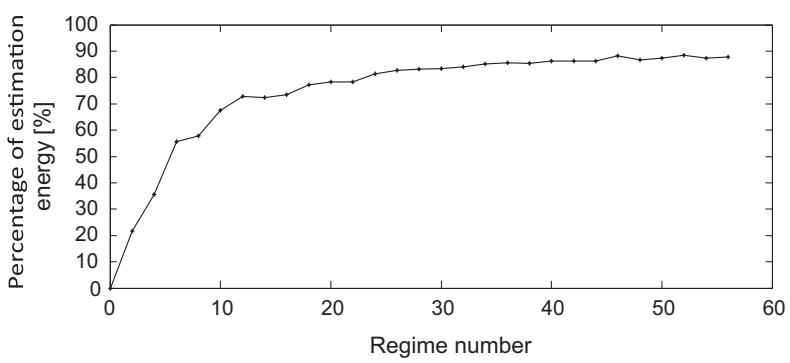

Fig. 7. (a) Order spectrum of the actual response and GSA estimates obtained with different speed resolutions and (b) percentage evolution of the GSA energy (with respect to the energy of the actual response) with the numbers of regimes for $10 \mathrm{~s}$ time record.

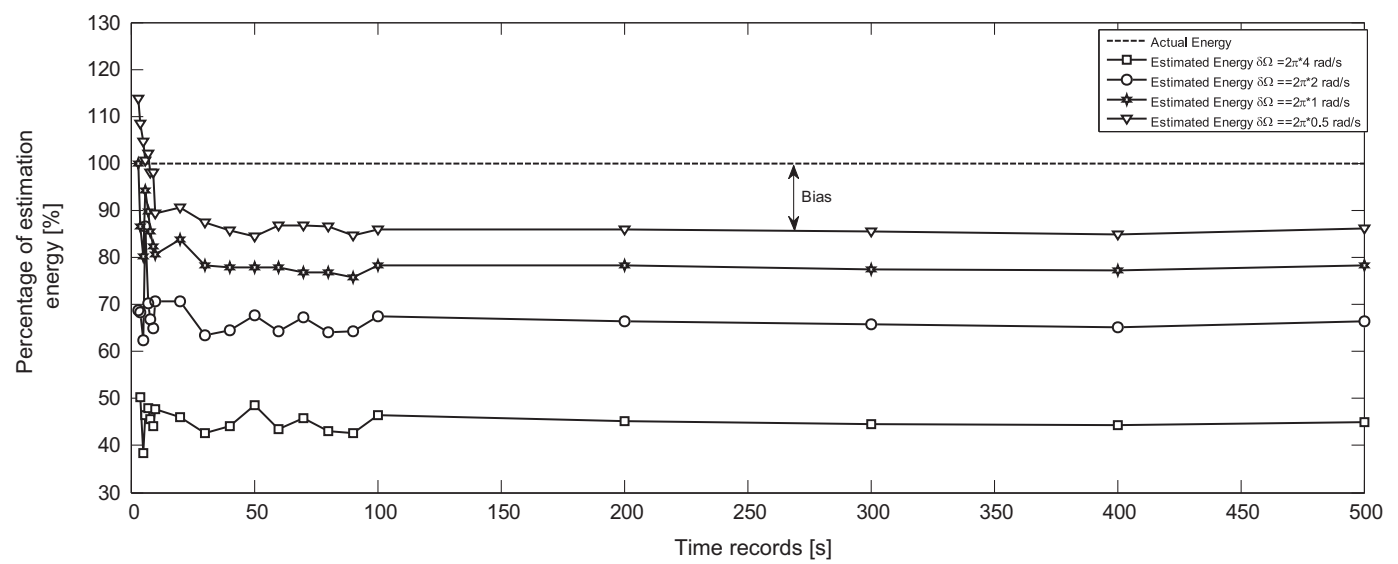

Fig. 8. Percentage evolution of the GSA energy (with respect to the energy of the actual response) with the number of regimes for $10 \mathrm{~s}$ time record.

\section{Experimental validation}

Similarly to the SA, the field of applications of the GSA covers multiple mechanical applications such as: machine diagnostics, separation of mechanical sources and identification of mechanical system [1]. This section reports a typical example in the field of rotating machine diagnostic, where deterministic gear related components contribute to the SES and mask the bearing fault signature-originally random. The aim is to show how to take advantage of the GSA to enhance the envelope-based diagnosis of rolling element bearings when operating under nonstationary condition. After a brief description of the test rig and experimental protocol, the concept of the GSA is demonstrated on gear vibration signals. Then, the GSA is used to extract the gear signal from its noisy environment. Last, the benefit of the separation is illustrated by analyzing the envelope of the residual signal in order to diagnose the bearings.

\subsection{Test rig and experimental protocol}

The test rig used in this paper is located at Cetim ${ }^{3}$ and illustrated in Fig. 9. It essentially comprises an asynchronous motor supplied by a variable-speed drive to control the motor speed, followed by a spur gear with 18 teeth and ratio of 1 . The gear is subjected to excessive wear. Two identical rolling element bearings are installed behind the spur gear; the healthy one (denoted as B1) is closer to the gears than the faulty one (denoted as B2) which is coupled to an alternator by means of a belt to impose a constant load. Bearing characteristics are listed in Table 1. An optical keyphasor of type "Brawn" is fixed close to the motor output to measure the rotational shaft position. In addition, two accelerometers, hereafter denoted as "Acc1" and "Acc2", are respectively branched on bearings B1 and B2 in the Z-direction to measure the produced vibrations. The sampling rate is set to $25.6 \mathrm{kHz}$.

First, experimental tests are conducted wherein five different constant speeds are imposed by the speed drive during $10 \mathrm{~s}$ in order to obtain five records for each accelerometer. Fig. 10 illustrates the raw signals measured by accelerometers Acc 1 and Acc2 at the chosen speeds 650, 950, 1250, 1550 and $1850 \mathrm{rev} / \mathrm{min}$. The importance of such an experiment is to obtain an

\footnotetext{
${ }^{3}$ Technical Center for Mechanical Industries-Senlis, France.
} 


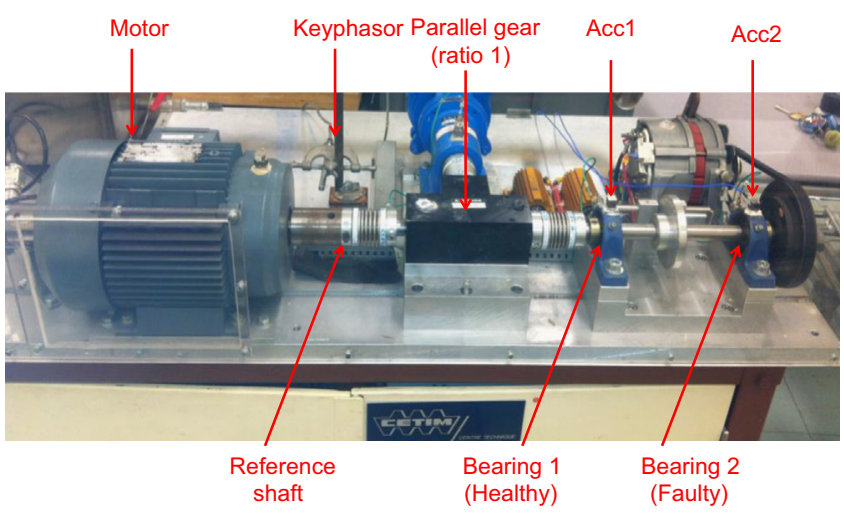

Fig. 9. The test rig located at CETIM.

Table 1

Characteristic of the rolling element bearing (Deep groove ball 6820).

\begin{tabular}{|c|c|c|c|c|c|c|c|}
\hline $\begin{array}{l}\text { Pitch diameter } \\
{[\mathrm{mm}]}\end{array}$ & $\begin{array}{l}\text { Rolling element } \\
\text { diameter }[\mathrm{mm}]\end{array}$ & $\begin{array}{l}\text { Number of roll- } \\
\text { ing elements }\end{array}$ & $\begin{array}{l}\text { Contact } \\
\text { angle }\end{array}$ & $\begin{array}{l}\text { Outer, housing, } \\
\text { diameter [mm] }\end{array}$ & $\begin{array}{l}\text { Inner, bore, dia- } \\
\text { meter [mm] }\end{array}$ & $\begin{array}{l}\text { Ball pass order- } \\
\text { outer race } \\
\text { (BPOO) }\end{array}$ & $\begin{array}{l}\text { Ball pass order- } \\
\text { inner race } \\
(\mathrm{BPOI})\end{array}$ \\
\hline 25 & 6 & 8 & 0.00 & 35 & 15 & 3.04 & 4.96 \\
\hline
\end{tabular}

actual reference of the machine behavior at the chosen operating speeds (which explains why they have been uniformly separated to systematically cover the machine operating margin). In the second experiment, a runup (from 500 to 2000 rev/ $\mathrm{min}$ ) and a random speed profile (between 500 and $2500 \mathrm{rev} / \mathrm{min}$ ) are imposed to the electric motor over $52 \mathrm{~s}$; the acquired acceleration signals are illustrated in Fig. 11 together with their corresponding speed profiles. Whereas the former type of speed profile is frequently met in rotor dynamics, the latter is for instance encountered in wind turbine applications.

\subsection{Validation of the GSA}

\subsubsection{Evaluation of GSA under different speed profiles}

By construction, the GSA theoretically returns the SA of a vibration signal at any operating speed independently of the speed profile in the record. The principal object of this section is to experimentally evidence this statement. For this purpose, the GSA is applied on variable speed records at some speeds, while the SA is applied on the constant-speed records at the same speeds. Since comparisons should be performed on estimations, the corresponding estimator errors (i.e. bias and variance) should be considered. Thus, the bias and the variance of the GSA should be theoretically calculated by means of the equations developed in Section 3.3, and used to build a threshold. The idea is to evaluate whether the difference between the GSA- and SA-based estimate are below the estimation threshold. If so, the aforementioned statement will be experimentally demonstrated.

To do so, the SA is applied to both acceleration signals on the constant-speed records of the first experiment (which is nothing else then the GSA applied to stepwise speed profile) in order to obtain a reference against which the GSA can be compared. Since the gears have a unit ratio, the reference period used in the average is set equal to the gear rotation, which makes possible to account for gear modulations. Next, the GSA is performed on the same accelerometer signal (Acc1) as in the case of (i) runup and (ii) random speed varying profiles. In order to evaluate the statistical performance of the estimator, it is proposed to compute the average values of the absolute bias and of the variance with respect to the SA. ${ }^{4}$ The average absolute bias at the operating speed $\omega_{r}$ is estimated by averaging the absolute value returned by Eq. (19) over the cycle duration, i.e.

$$
\widehat{b}\left(\omega_{r}\right)=\frac{1}{\Theta} \int_{0}^{\Theta}\left|\operatorname{Bias}\left\{\widehat{m}_{Y}\left(\bar{\theta}, \omega_{r}\right)\right\}\right| \mathrm{d} \bar{\theta},
$$

whereas the average variance is estimated by averaging the value returned by Eq. (20) over the cycle duration, i.e.

$$
\widehat{\sigma}^{2}\left(\omega_{r}\right)=\frac{1}{\Theta} \int_{0}^{\Theta}\left|\operatorname{Var}\left\{\widehat{m}_{Y}\left(\bar{\theta}, \omega_{r}\right)\right\}\right| \mathrm{d} \bar{\theta}
$$

\footnotetext{
${ }^{4}$ The distributions $\left.m_{Y} \top \theta, \omega_{r}\right)$ and $\left.\left.P_{Y}\right\rceil \theta, \omega_{r}\right)$ are returned by the SAs of the signals and of the residual powers, respectively, applied on the constantspeed records of the first experiment.
} 
(a)

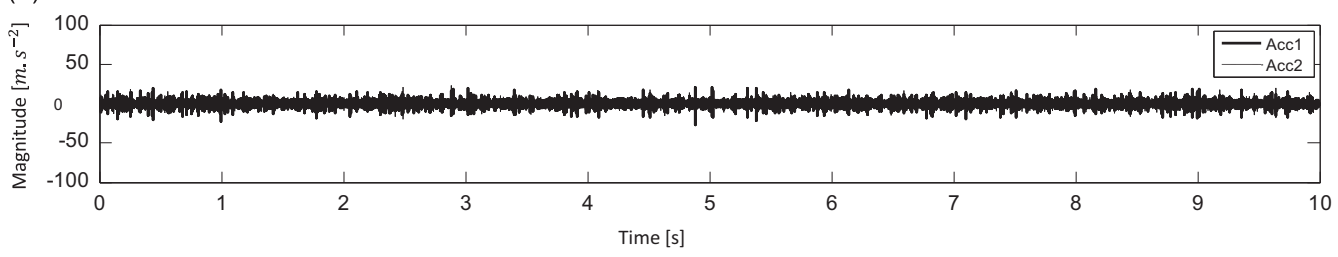

(b)

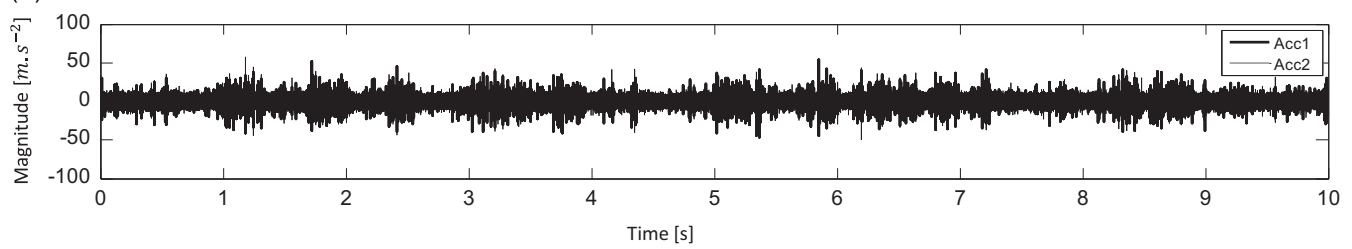

(c)

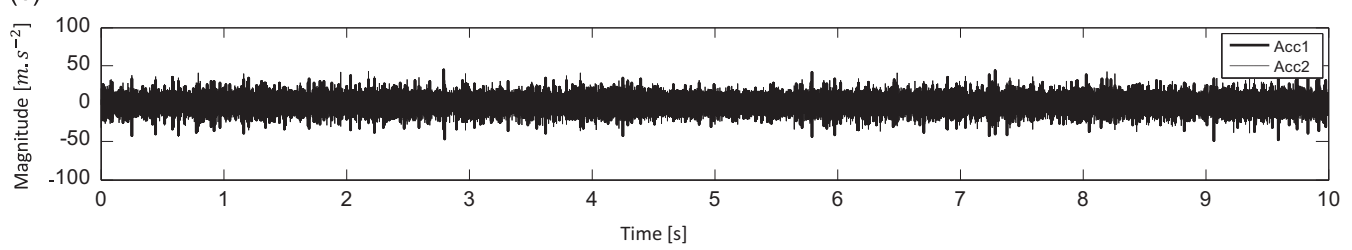

(d)

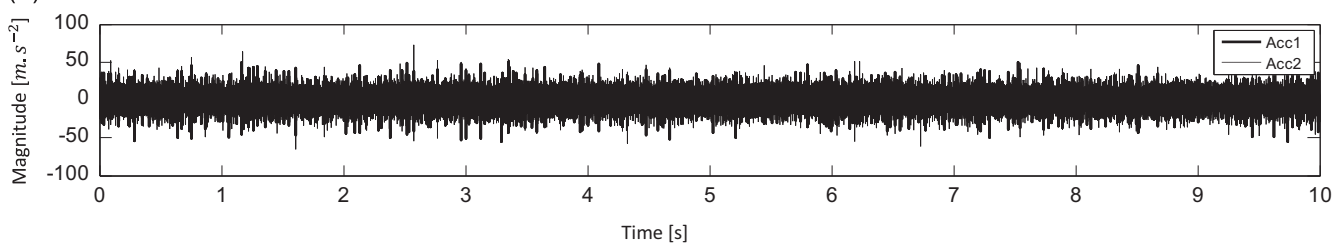

(e)

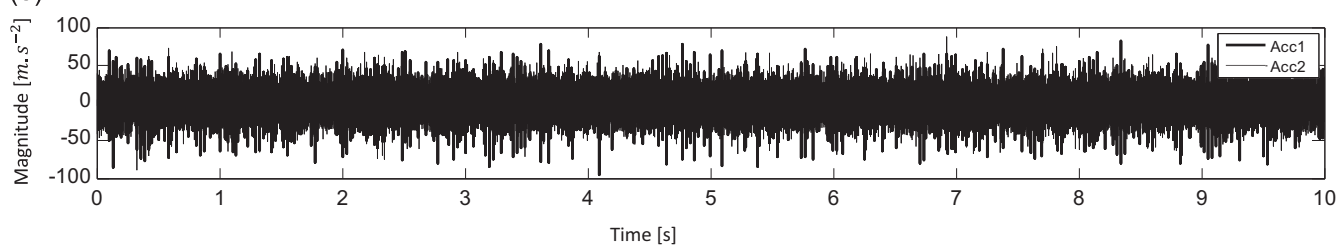

Fig. 10. (a) Acceleration signals acquired at operating speeds (a) $650 \mathrm{rev} / \mathrm{min}$, (b) $950 \mathrm{rev} / \mathrm{min}$, (c) $1250 \mathrm{rev} / \mathrm{min}$, (d) $1550 \mathrm{rev} / \mathrm{min}$ and (e) $1850 \mathrm{rev} / \mathrm{min}$.

Having an estimate of the average bias and variance, the level of the estimation error can be evaluated as

$$
\text { thres }=\widehat{b}\left(\omega_{r}\right)+3 \cdot \widehat{\sigma}\left(\omega_{r}\right) \text {. }
$$

In the runup case, the comparisons between the GSA estimates and the SA-based references are displayed in Fig. 12 for the shaft speeds (a) 650, (b) 950, (c) 1250, (d) 1550 and (e) $1850 \mathrm{rev/min} \mathrm{(the} \mathrm{speeds} \mathrm{under} \mathrm{which} \mathrm{the} \mathrm{first} \mathrm{experiment} \mathrm{has}$ been conducted); the absolute value of the corresponding estimation errors are illustrated in (f)-(j) together with the calculated thresholds. Results for the case of varying speed profile are reported in Fig. 13. In general, the similarity between the estimated signatures is remarkable for both cases: the estimation error mostly stays below the threshold at the exception of some phase mismatch at $1250 \mathrm{rev} / \mathrm{min}$ (see the third raw in Figs. 12 and 13). This confirms the model given in Eq. (9). Also, since the estimation performance does not show dependence on the speed profile (runup or random variable speed), one can claim that signal statistics do not depend on higher derivatives of $\theta$, which validates the assumption leading to Eq. (7). It is worth noting that, despite being below the threshold, the estimation errors of the regimes $650 \mathrm{rev} / \mathrm{min}$ (see Figs. 12(f) and 13(f)) and $1850 \mathrm{rev/min} \mathrm{(see} \mathrm{Figs.} \mathrm{12(j)} \mathrm{and} \mathrm{13(j))} \mathrm{are} \mathrm{high.} \mathrm{According} \mathrm{to} \mathrm{Eq.} \mathrm{(20),} \mathrm{the} \mathrm{high} \mathrm{error} \mathrm{in} \mathrm{the} \mathrm{former}$ is due to the low number of cycles associated with the regime, while the high error in the second is due to the high mean instantaneous power.

Next, the GSA applied on runup and random speed profiles are compared in Fig. 14. The similarity of the compared plots indicates the invariance of the process properties (i.e. the CNS field) whatever the speed trajectory. Last, the GSA is applied 
(a)

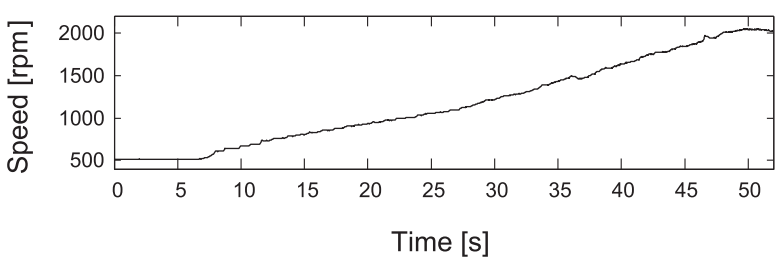

(c)

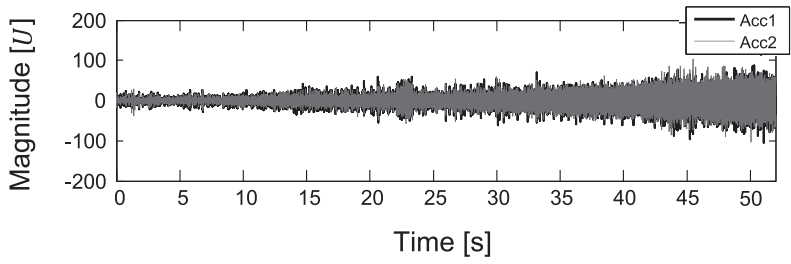

(b)

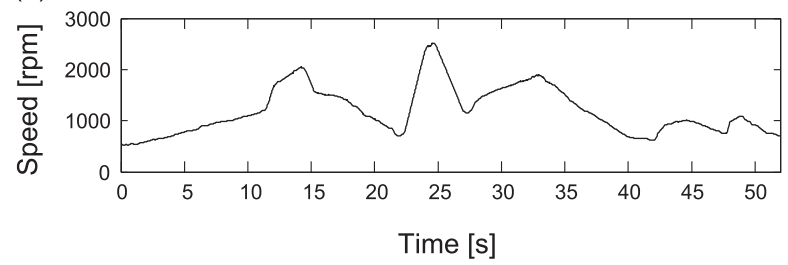

(d)

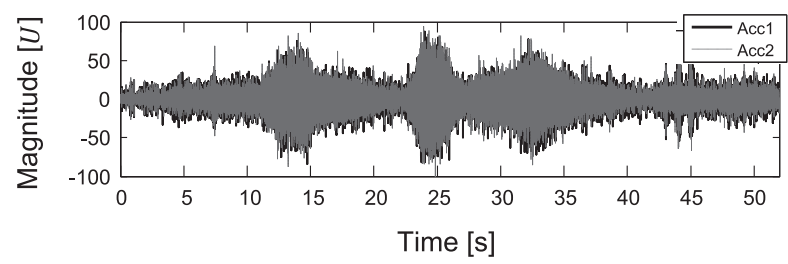

Fig. 11. (a) Runup speed profile and (b) its corresponding acceleration signals (Acc1 and Acc2). (c) Random speed profile and (d) its corresponding acceleration signals (Acc1 and Acc2).

(a)

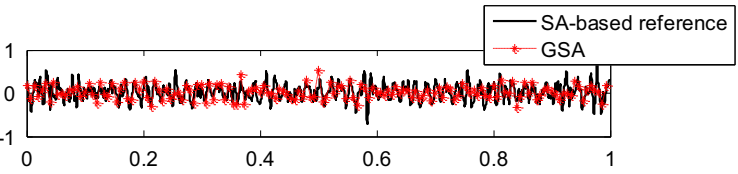

(b)

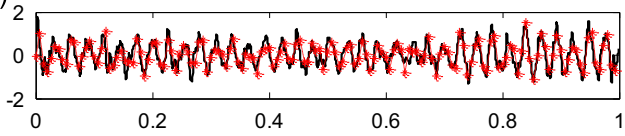

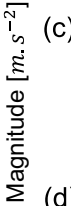

(d)

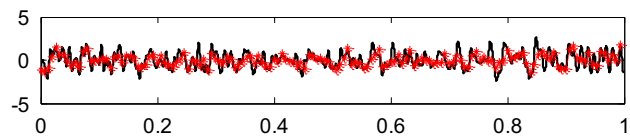

(e)

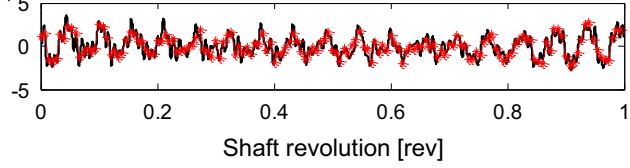

(f)

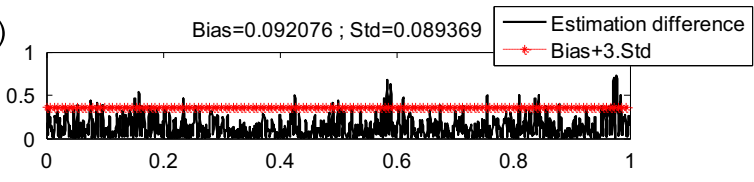

(g)

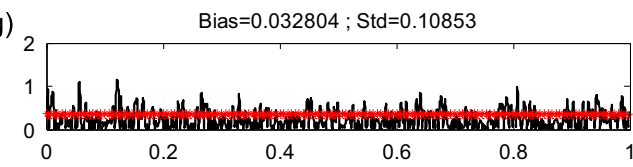

is (h)

Bias $=0.067231 ;$ Std $=0.14055$

ह

(i)

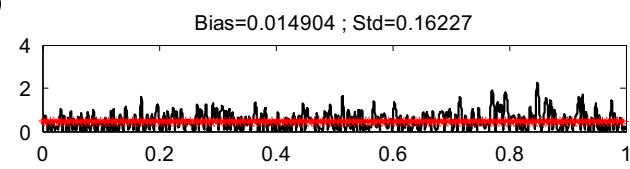

(j)

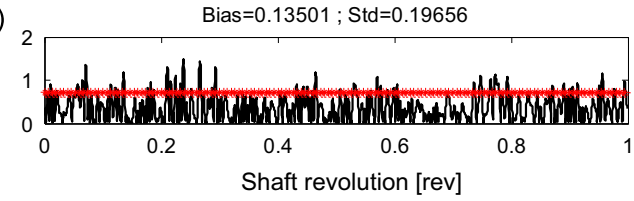

Fig. 12. Comparison between the SA of Acc1 signal acquired at constant speeds and the GSA of the same accelerometer acquired in runup: (a) 650 rev/min, (b) $950 \mathrm{rev} / \mathrm{min}$, (c) $1250 \mathrm{rev} / \mathrm{min}$, (d) $1550 \mathrm{rev} / \mathrm{min}$, (e) $1850 \mathrm{rev} / \mathrm{min}$; (f)-(j) are the absolute differences of the plots in (a)-(e) respectively and their corresponding threshold.

on signal Acc1 of the runup speed profile (Fig. 11(b)) together with the classical SA. Results are displayed in Fig. 15 wherein the same unit scale is used to evidence the amplitude differences. As expected from the theory, the deterministic component varies with speed in terms of phase and energy (see Fig. 15(b-f)) which results in an attenuation of the SA magnitude (see Fig. 15(a)).

\subsubsection{Extraction of the gear contribution}

In the following, the efficiency of the GSA in extracting the deterministic component is investigated. For this purpose, the GSA is applied on vibration signals produced under runup and random regimes in order to extract the deterministic gear related components under both cases. The GSA estimates of signals Acc1 and Acc2 in runup computed with 20 nonoverlapped intervals are displayed in Fig. 16(a) and (d) runup and the corresponding amplitude order spectra are displayed 

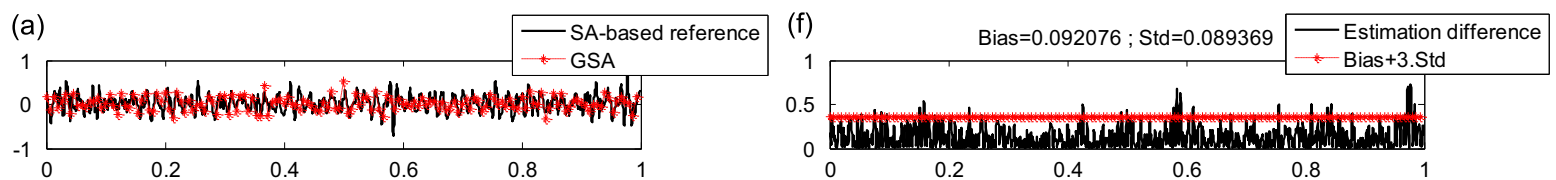

(b)

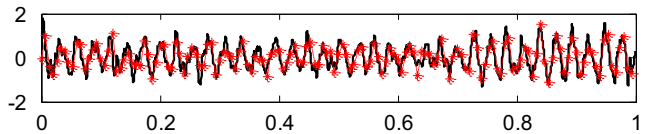

(g)
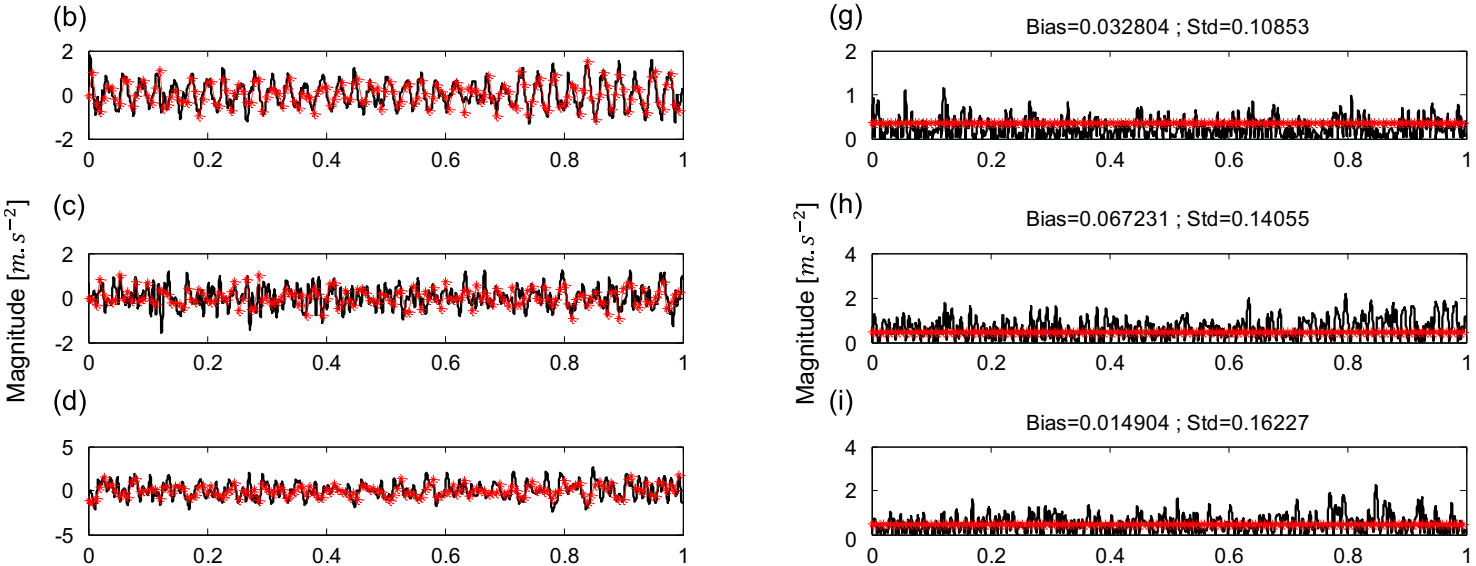

(e)

(j)
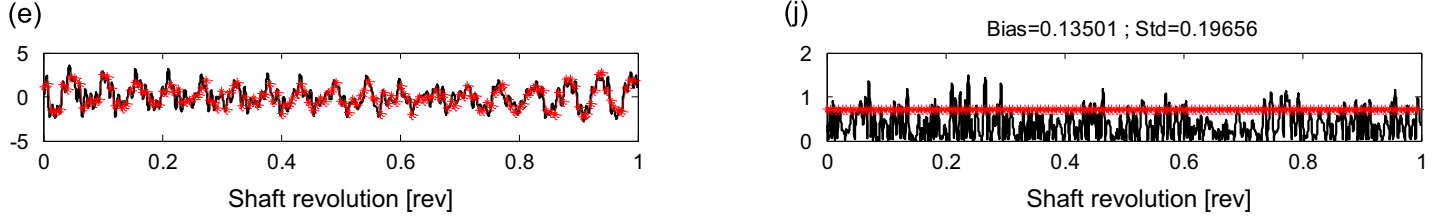

Fig. 13. Comparison between the SA of signal Acc1 acquired at constant speeds and the GSA of the same accelerometer acquired in random variable speed: (a) $650 \mathrm{rev} / \mathrm{min}$, (b) $950 \mathrm{rev} / \mathrm{min}$, (c) $1250 \mathrm{rev} / \mathrm{min}$, (d) $1550 \mathrm{rev} / \mathrm{min}$, (e) $1850 \mathrm{rev} / \mathrm{min}$; (f)-(j) are the absolute differences of the plots in (a)-(e) respectively and their corresponding threshold.

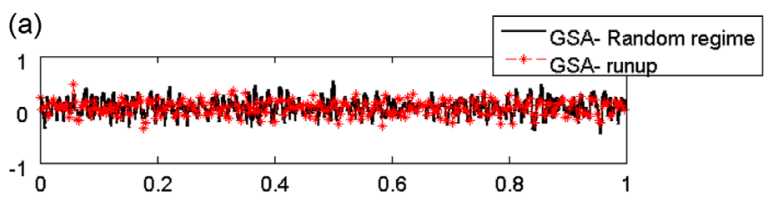

(b)
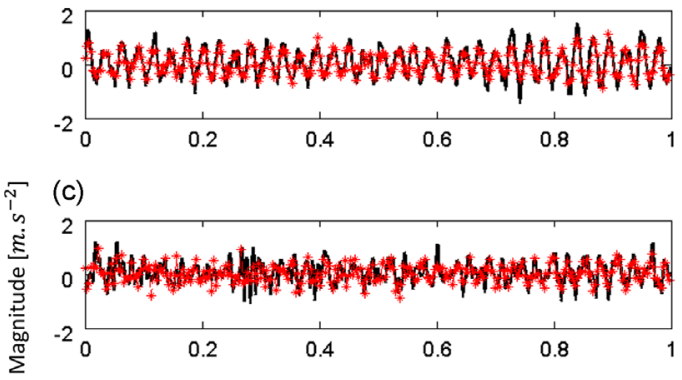

(d)

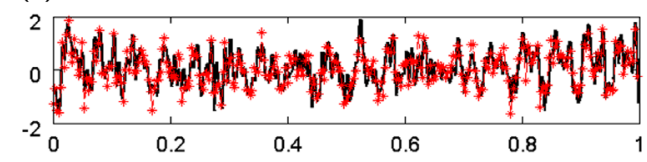

(e)

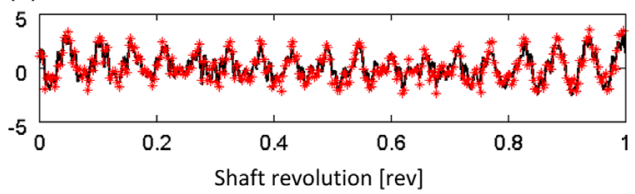

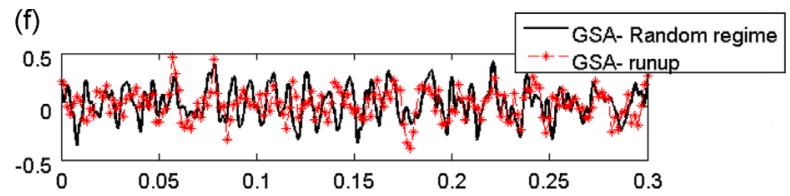

(g)
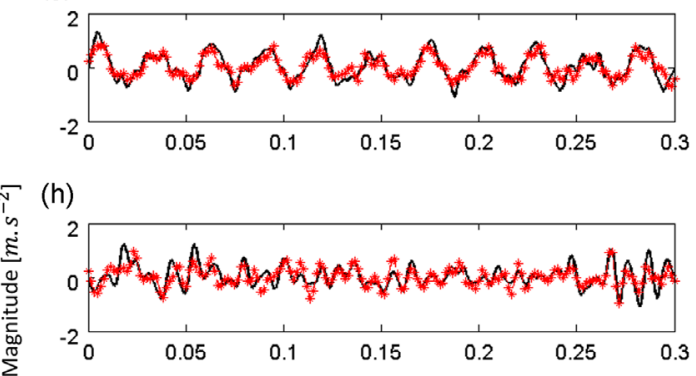

(i)

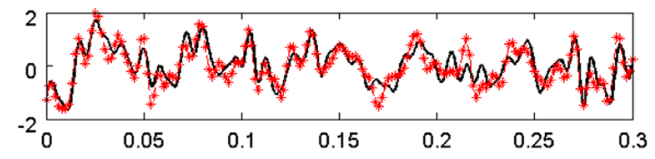

(j)

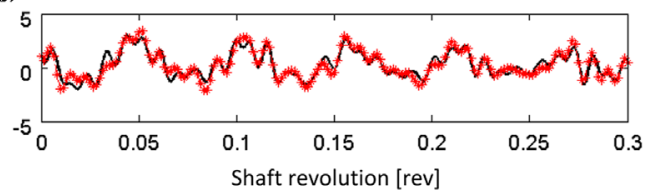

Fig. 14. Comparison between the GSA of signal Acc1 acquired at runup and the GSA of the same accelerometer acquired at random variable speed:

(a) $650 \mathrm{rev} / \mathrm{min}$, (b) $950 \mathrm{rev} / \mathrm{min}$, (c) $1250 \mathrm{rev} / \mathrm{min}$, (d) $1550 \mathrm{rev} / \mathrm{min}$, (e) $1850 \mathrm{rev} / \mathrm{min}$; (f)-(j) are the close-ups between 0 and $0.3 \mathrm{rev}$ of plots (a)(e) respectively. 
(a)

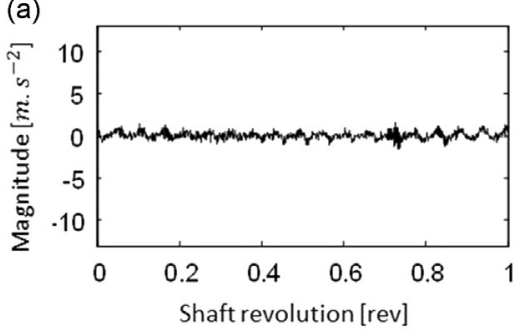

(d)

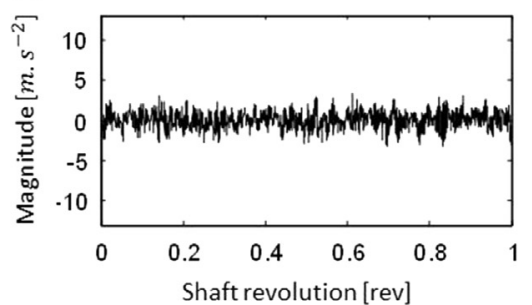

(b)

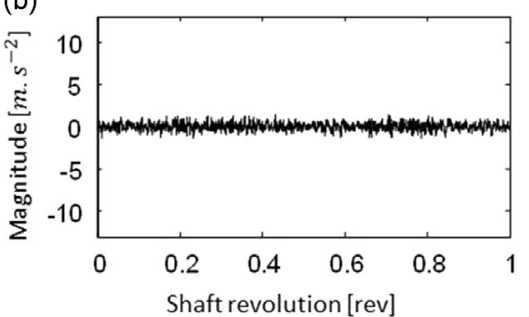

(e)

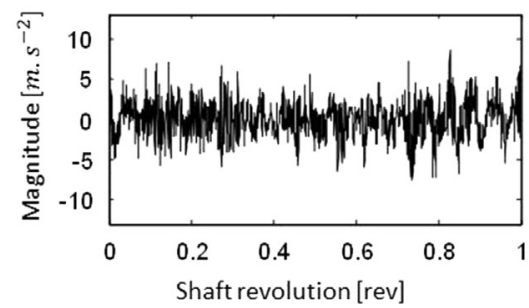

(c)

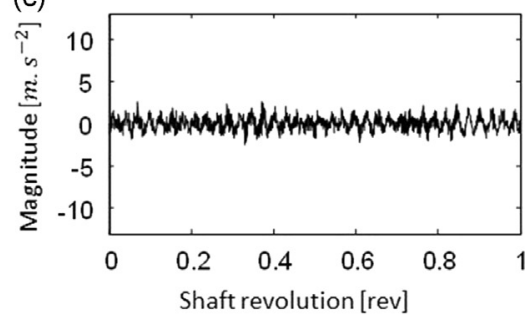

(f)

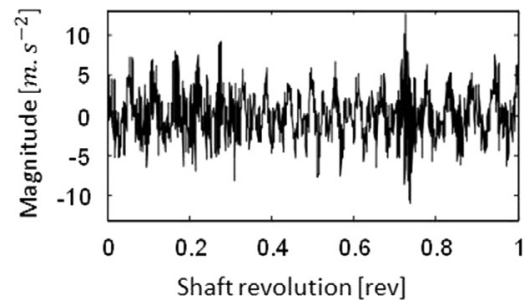

Fig. 15. Classical SA (a) and GSA applied on signal Acc1 in runup speed profile for the speeds (b) $650 \mathrm{rev} / \mathrm{min}$ (c), $950 \mathrm{rev} / \mathrm{min}$ (d), $1250 \mathrm{rev} / \mathrm{min}$ (e), $1550 \mathrm{rev} / \mathrm{min}$ and (f) $1850 \mathrm{rev} / \mathrm{min}$. The same unit scale is used to evidence the differences.

(a)

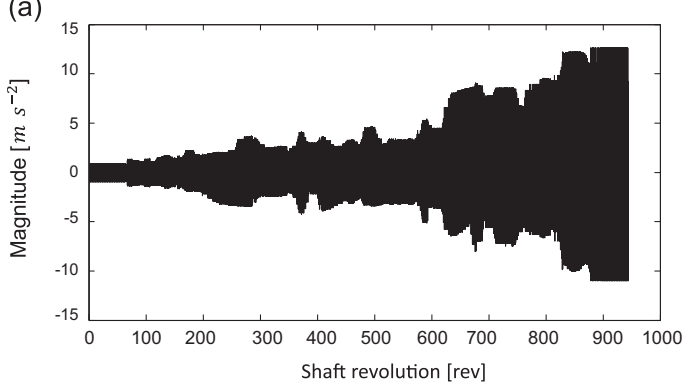

(b)

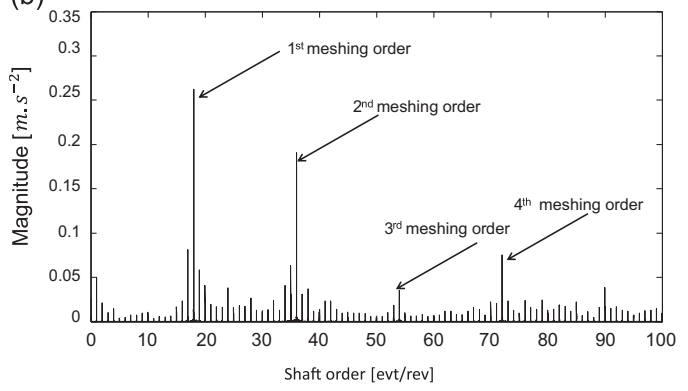

(c)

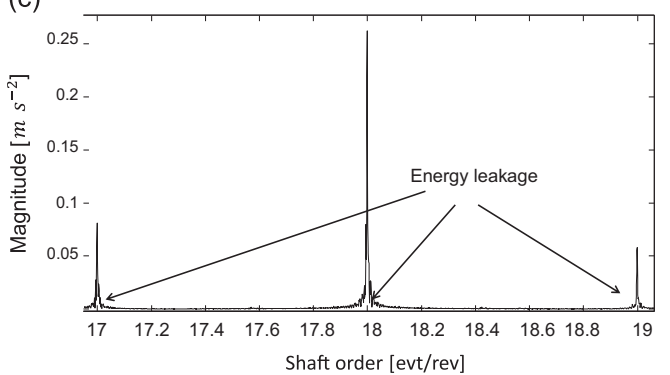

(d)

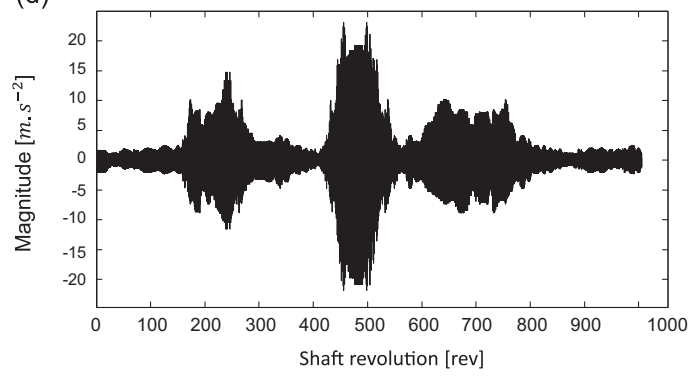

(e)

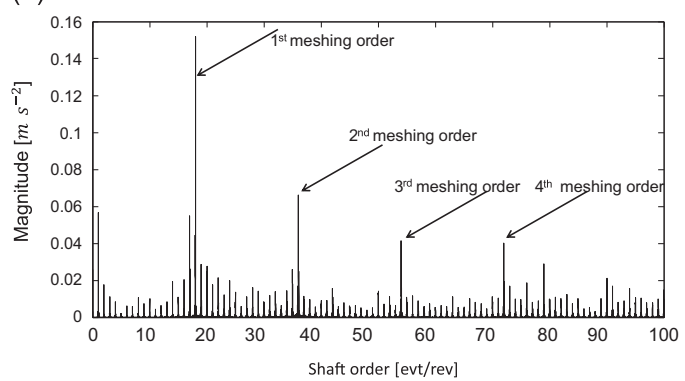

(f)

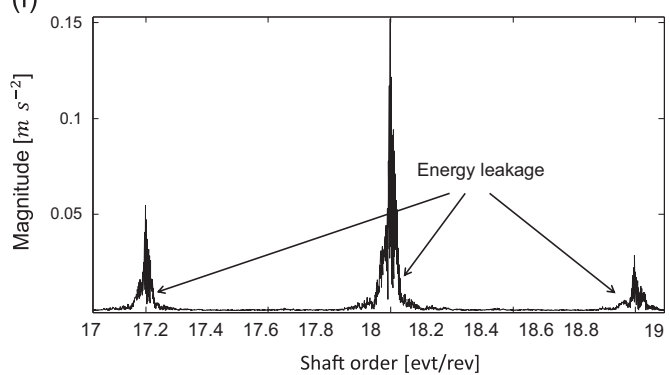

Fig. 16. (a) GSA of signal Acc1 in runup speed regime estimated with 20 non-overlapping intervals, (b) its amplitude order spectrum and (c) its zoom around the 1st meshing order. (d) GSA estimate of signal Acc2 in random speed regime estimated with 20 non-overlapping intervals, (e) its amplitude order spectrum and (f) its zoom around the 1st meshing order. 
in (b) and (e), respectively. Observing its evolution in the angular domain (see Fig. 16(a) and (d)), the GSA is able to track the energy variations of the deterministic component.

Observing its order-domain counterpart (see Fig. 16(a) and (d)), the GSA seems to have a similar behavior of the SA: it presents equally spaced harmonics as if a comb filter was applied. Yet, when checking these harmonics (see Fig. 16(c) and (f)), one can clearly notice the energy leakage around the considered orders. This actually means that-in some sense-the GSA has tracked the (amplitude and phase) distortions due to the TF of the system. It is worth noting that the energy leakage around the harmonics is dependent on the speed variability in the record. The higher this variability is, the higher the induced leakage. This can be simply checked by comparing the broadening of the harmonics in Fig. 16(c) and (f). The width of the harmonics is wider for the random regime than for the runup because speed variability is greater in the former case.

In order to consolidate the GSA results, the next step is to investigate the residual signal obtained from subtracting the GSA estimate from the original signal. In the case of a perfect estimation, the broadened harmonics related to the deterministic component should be absent from the residues. For this purpose, the amplitude order spectrum of the SA and GSA residues signals are compared in Fig. 17 for Acc2 signal in runup regime and Acc1 signal in random speed-varying regime. As expected, the SA fails in both cases to eliminate the deterministic component wherein a significant part of the harmonic still exists. In details, the SA is equivalent to applying a narrow-band comb filter spaced with the machine order, thus it is unable to entirely extract the broadened harmonics as they fall outside the lobes of the comb filter. Therefore, once can see a local fall at all the meshing order harmonics in all the plots of Fig. 17. Interestingly, the GSA evidences almost total suppression, thus validating its efficiency in extracting the deterministic component in variable speed conditions.

(a)

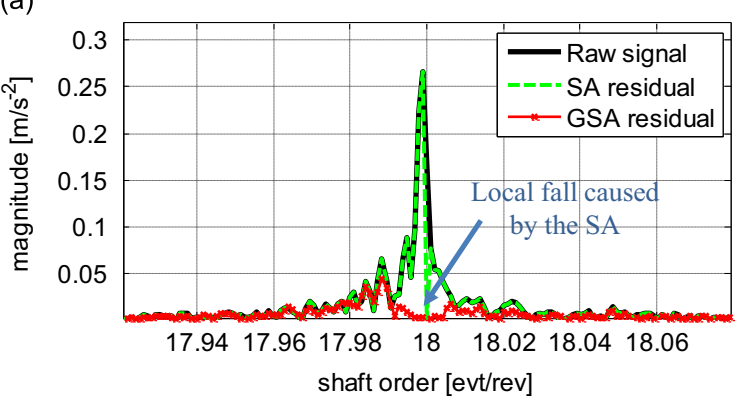

(b)

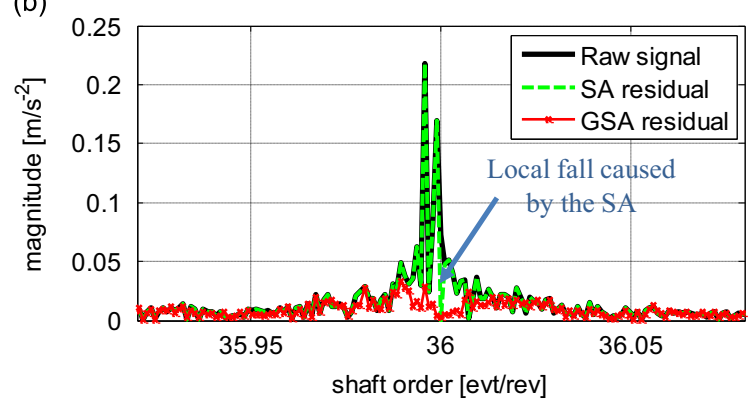

(c)

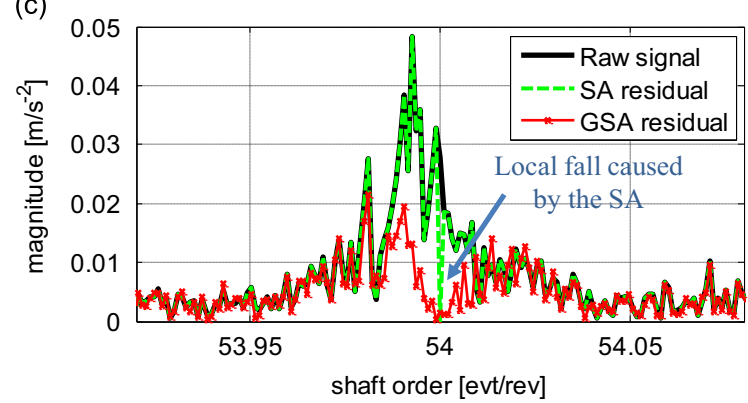

(d)

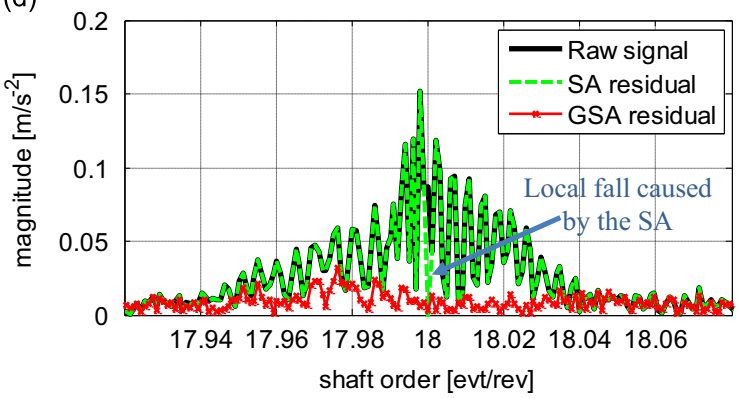

(e)

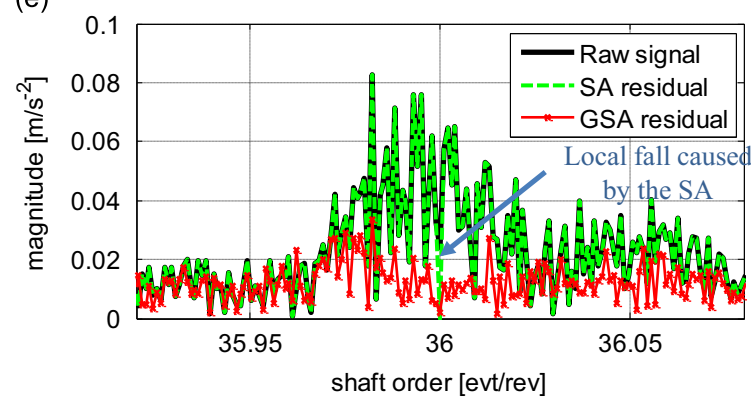

(f)

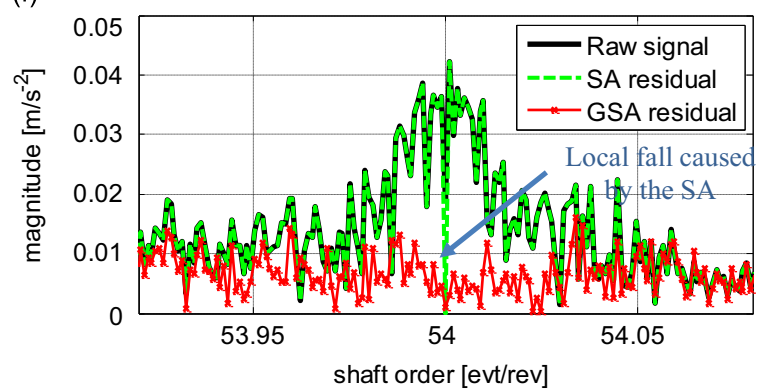

Fig. 17. Amplitude order spectrum of the SA and GSA residues of Acc 2 in runup speed regime, close-ups around (a) the 1 st meshing harmonic, (b) the 2 nd meshing harmonic and (c) the 3rd meshing harmonic. Amplitude order spectrum of the SA and GSA residues of Acc1 signal in random speed-varying regime, close-ups around (d) the 1st meshing harmonic, (e) the 2nd meshing harmonic and (f) the 3rd meshing harmonic. 


\subsection{Envelope enhancement}

In vibration analysis of rotating machine, the squared envelope spectrum (SES) is one of the most efficient indicators for the assessment of CS2 sources which are typical symptoms of damage in rolling element bearing faults [35]. Similarly to other CS2 tools, the SES should be applied on the pure random part of the signal; otherwise spurious harmonics may appear and hide the actual second-order content. In speed varying regimes, the SES is combined with computed order tracking to obtain an order-domain representation. This section reports a typical issue in the field of rotating machine diagnostic, where deterministic gear related components contribute to the SES and mask the bearing fault signature-originally random. The principal object is to investigate the benefits brought by the GSA in enhancing the envelope analysis for bearing fault detection.

For this purpose, Acc2 signal in runup speed regime is picked up to evaluate the signature of the faulty bearing. Next, after angular resampling, the SES is applied on the raw signal over the entire order band, on the SA residue and on the GSA residue as shown in the diagram of Fig. 18. The obtained results are reported in Fig. 19. As expected, the SES of the raw signal evidences several misleading harmonics synchronous with the reference shaft order, whereas slight enhancement is perceived after removing the SA (see Fig. 19(a) and (b)). Noticeably, the SES of the residual signal after removing the GSA much better evidences the bearing signature (see Fig. 19(c)), thus demonstrating the utility of the introduced technique for envelope based vibration analysis.

\section{Conclusion}

The object of this paper was to extent the synchronous average, traditionally applied to cyclostationary signals, to the general class of cyclo-non-stationary (CNS) signals wherein the operating speed is allowed to be nonstationary. It has been shown that, in the case of a system operating under variable speed, cyclo-non-stationarity can be produced by the transfer function from the excitation to the accelerometer. This phenomenon induces systematic changes in the signal phase and magnitude, thus changing its structure. These changes have been modeled by means of an explicit dependence on the speed. As a consequence, the deterministic part loses its (angle-) periodicity, thus jeopardizing the synchronous average.

Therefore, a novel quantity coined the "generalized synchronous average (GSA)" has been introduced to extract the deterministic part of a CNS signal. This quantity, when evaluated at an arbitrary speed, has the advantage of expressing the mean value of the signal as if the system was operating steadily at that speed. Two estimators of the GSA have been proposed. The first one returns the synchronous average of the signal at predefined discrete operating speeds. A brief statistical study of it is performed, aiming to provide the user with confidence intervals that reflect the "quality" of the estimator according to the SNR and the estimated speed. The second estimator returns a smoothed version of the former by enforcing continuity over the speed axis. It helps to reconstruct the deterministic component by tracking a specific trajectory dictated by the speed profile (assumed to be known a priori). The proposed approach has been tested on an actual benchmark comprising a typical powertrain. The aim was twofold: first to validate the proposed CNS model and the GSA estimators and second to show the usefulness of the proposed approach for rolling element bearing diagnostics in variable operating speed. Interestingly, the GSA proved efficient in estimating the deterministic gear contribution, showing considerable enhancement over the classical SA.

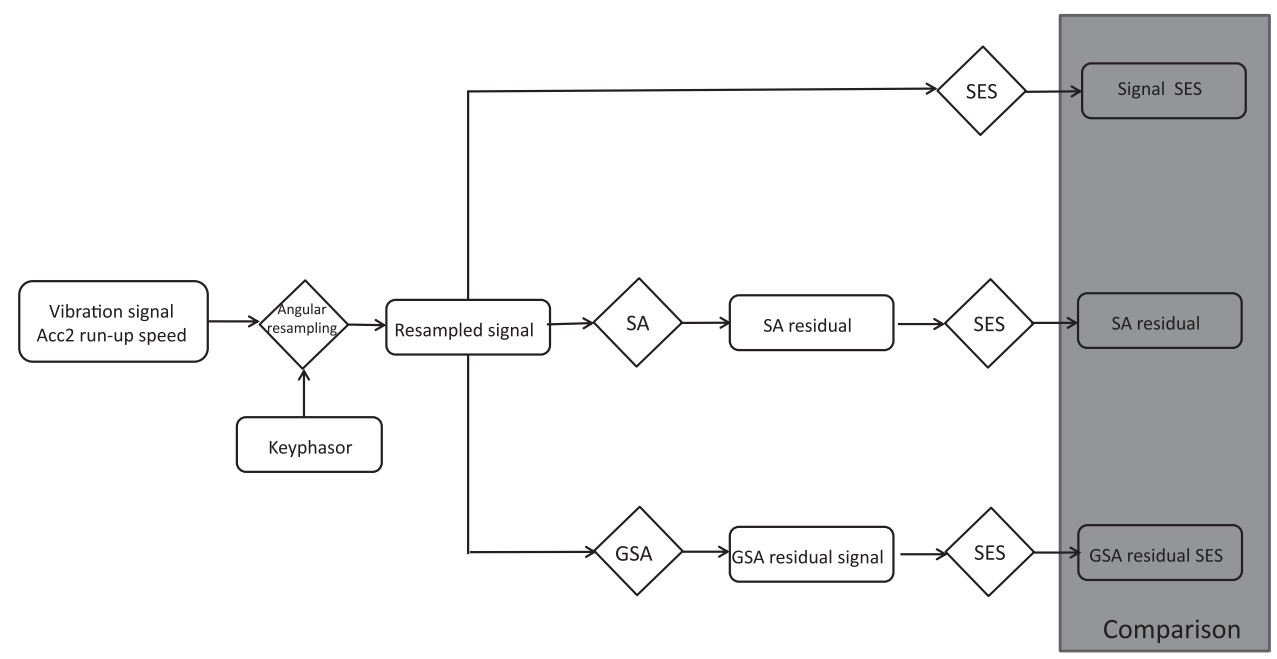

Fig. 18. Comparison of the SES of residue, the SES of SA residue and the SES of the GSA residue. 
(a)

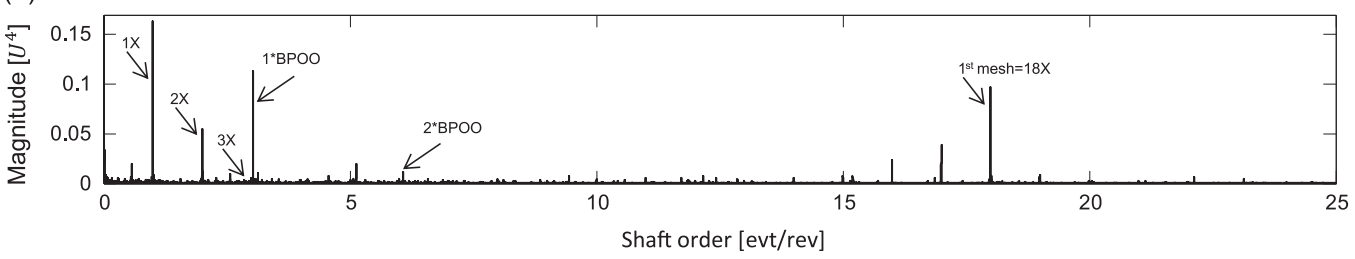

(b)

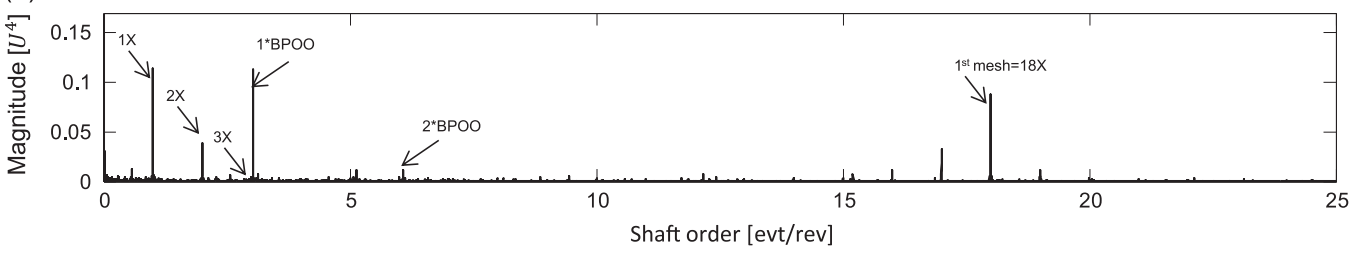

(c)

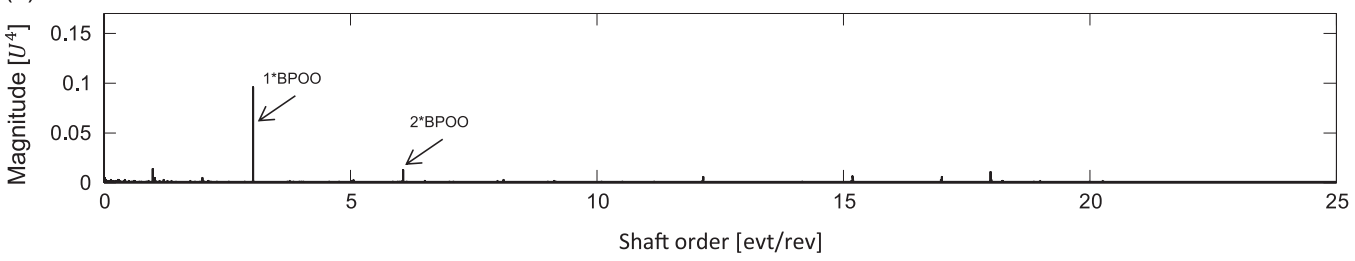

Fig. 19. SES of the (a) resampled signal, (b) the SA residue and (c) the GSA residue applied on signal Acc2 in runup.

Limitations of the GSA are inherently related to estimation issues. In particular, it has been assumed that the speed variations are slower than the signal cycle; although this condition seems reasonable in several applications, it can easily be relaxed in the general case by averaging portions of signal smaller than cycles. Another issue concerns the setting of the estimation parameters such as the speed resolution, the number of regimes, and the repartition of the central frequencies. In the present paper, these settings have been governed by simplicity. For example, the regimes were chosen to be uniformly distributed along the speed axis. This actually makes the estimation variance dependent on the operating speed; again, this could easily be alleviated by allowing the speed resolution to be inversely proportional to the speed probability distribution.

Eventually, authors believe that the GSA can be also used for estimating second-order quantities such as instantaneous autocorrelation or the spectral correlation as functions of speed. As well, it can be applied to other domains such as gear diagnostics, noise separation in IC engines, etc.

\section{Acknowledgments}

This work was conducted in the framework of the LabEx CeLyA ("Centre Lyonnais d'Acoustique", ANR-10-LABX-60).

\section{Appendix}

\section{a) Proof of Eq. (19).}

Let us now apply the statistical expectation to the raw estimator of the GSA applied to the model in Eq. (8)

$$
\mathbb{E}\left\{\widehat{m}_{Y}\left(\bar{\theta}, \omega_{r}\right)\right\}=\mathbb{E}\left\{\frac{1}{\operatorname{card}\left\{K_{r}\right\}} \sum_{k \in K_{r}}\left[\sum_{\alpha} c_{Y}^{\alpha}(\bar{\theta}+k \Theta, \omega(\bar{\theta}+k \Theta)) \mathrm{e}^{\left.\mathrm{j} 2 \pi \alpha \frac{(\bar{\theta}+k \theta)}{\Theta}\right]}\right\} .\right.
$$

By exploiting the linearity of the expectation operator

$$
\mathbb{E}\left\{\widehat{m}_{Y}\left(\bar{\theta}, \omega_{r}\right)\right\}=\frac{1}{\operatorname{card}\left\{K_{r}\right\}} \sum_{k \in K_{r}}\left[\sum_{\alpha} \bar{c}_{Y}^{\alpha}(\omega(\bar{\theta}+k \Theta)) \mathrm{e}^{\mathrm{j} 2 \pi \alpha \frac{(\bar{\theta}+k \theta)}{\theta}}\right] .
$$


According to Slutsky's theorem on probability limits, the sample averaging operator $\frac{1}{\operatorname{card}\left\{K_{r}\right\}} \sum_{k \in K_{r}}\{*\}$ converges to the statistical expectation $\mathbb{E}_{\omega}\{*\}$ with respect to the distribution of $\omega(\bar{\theta}+k \Theta)$ in the interval $\left[\omega_{r}-\frac{\delta \omega}{2} ; \omega_{r}-\frac{\delta \omega}{2}\right]$, i.e.

$$
\mathbb{E}\left\{\widehat{m}_{Y}\left(\bar{\theta}, \omega_{r}\right)\right\}=\mathbb{E}_{\omega}\left\{\sum_{\alpha} \bar{c}_{Y}^{\alpha}(\omega) \mathrm{e}^{\mathrm{j} 2 \pi \alpha_{\bar{\theta}}^{\bar{\theta}}}\right\}=\mathbb{E}_{\omega}\left\{m_{Y}(\bar{\theta}, \omega)\right\} .
$$

The rest of the proof follows by approximating the distribution of $\omega$ by a uniform law in the interval $\left[\omega_{r}-\delta \omega / 2 ; \omega_{r}+\delta \omega / 2\right]$ and after performing a second-order Taylor approximation around $\omega_{r}$.

a) Proof of Eq. (20).

Similarly to the previous proof, the variance is

$$
\operatorname{Var}\left\{\widehat{m}_{Y}\left(\bar{\theta}, \omega_{r}\right)\right\}=\operatorname{Var}\left\{\frac{1}{\operatorname{card}\left\{K_{r}\right\}} \sum_{k \in K_{r}}\left[\sum_{\alpha} c_{Y}^{\alpha}(\bar{\theta}+k \Theta, \omega(\bar{\theta}+k \Theta)) \mathrm{e}^{\mathrm{j} 2 \pi \alpha \frac{(\bar{\theta}+k \theta)}{\theta}}\right]\right\} .
$$

Since terms in the sum do not overlap

$$
\operatorname{Var}\left\{\widehat{m}_{Y}\left(\bar{\theta}, \omega_{r}\right)\right\}=\frac{1}{\left(\operatorname{card}\left\{K_{r}\right\}\right)^{2}} \sum_{k \in K_{r}} P_{Y}((\bar{\theta}+k \Theta), \omega(\bar{\theta}+k \Theta))
$$

where $P_{Y}(\bar{\theta}, \omega)=\operatorname{Var}\left\{\sum_{\alpha} c_{Y}^{\alpha}(\bar{\theta}, \omega) \mathrm{e}^{\mathrm{j} 2 \pi \alpha^{\bar{\theta}}}\right\}$ is the instantaneous power of the residue (i.e. purely random) that depends on the speed. According to Slutsky's theorem

$$
\operatorname{Var}\left\{\widehat{m}_{Y}\left(\bar{\theta}, \omega_{r}\right)\right\}=\frac{1}{\operatorname{card}\left\{K_{r}\right\}} \mathbb{E}_{\omega}\left\{P_{Y}(\bar{\theta}, \omega)\right\}
$$

The rest of the proof follows by approximating the distribution of $\omega$ by a uniform law and performing a second-order Taylor approximation around $\omega_{r}$.

\section{References}

[1] J. Antoni, Cyclostationary by examples, Mechanical Systems and Signal Processing 23 (4) (2009) 987-1036.

[2] J. Antoni, R.B. Randall, Differential diagnosis of gear and bearing faults, Journal of Sound and Vibration 124 (2) (2002) $165-171$.

[3] J. Antoni, R.B. Randall, Unsupervised noise cancellation for vibration signals: Part II-a novel frequency-domain algorithm, Mechanical Systems and Signal Processing 18 (2004) 103-117.

[4] R.B. Randall, Vibration-Based Condition Monitoring: Industrial, Automotive and Aerospace Applications, John Wiley and Sons, West Sussex, 2011.

[5] S. Braun, The extraction of periodic waveforms by time domain averaging, Acustica 32 (1975) 69-77.

[6] P.D. McFadden, M. Toozhy, Application of synchronous averaging to vibration monitoring of rolling element bearing, Mechanical Systems and Signal Processing 14 (2000) 891-896.

[7] K.R. Fyfe, E.D.S. Munck, Analysis of computed order tracking, Mechanical Systems and Signal Processing 11 (2) (1997) 187-205.

[8] P.D. McFadden, Interpolation techniques for time domain averaging of gear vibration, Mechanical Systems and Signal Processing 3 (1) (1989) 87-97.

[9] F. Bonnardot, M. ElBadaoui, R.B. Randall, J. Daniere, F. Guillet, Use of the acceleration signal of a gearbox in order to perform angular resampling (with limited speed fluctuation), Mechanical Systems and Signal Processing 19 (2005) 766-785.

[10] J. Antoni, F. Bonnardot, A. Raad, M. El Badaoui, Cyclostationary modelling of rotating machine vibration signals, Mechanical Systems and Signal Processing 18 (2004) 1285-1314.

[11] C.J. Stander, P.S. Heyns, Transmission path phase compensation for gear monitoring under fluctuating load conditions, Mechanical Systems and Signal Processing 20 (7) (2006) 1511-1522.

[12] P. Borghesani, P. Pennacchi, R.B. Randall, R. Ricci, Order tracking for discrete-random separation in variable speed conditions, Mechanical Systems and Signal Processing 30 (2012) 1-22.

[13] J. Antoni, N. Ducleaux, G. NGhiem, S. Wang, Separation of combustion noise in IC engines under cyclo-non-stationary regime, Mechanical Systems and Signal Processing 38 (1) (2009) 223-236.

[14] M.D. Coats, N. Sawalhi, R.B. Randall, Extraction of tacho information from a vibration signal for improved synchronous averaging, Proceedings of Acoustics (2009).

[15] Z. Daher, E. Sekko, J. Antoni, C. Capdessus, L. Allam, Estimation of the synchronous average under varying rotating speed condition for vibration monitoring, Proceedings of ISMA (2010).

[16] J. Urbanek, T. Barszcz, J. Antoni, A two-step procedure for estimation of instantaneous rotational speed with large fluctuations, Mechanical Systems and Signal Processing 38 (1) (2013) 96-102.

[17] D.A. Wallace, M.S. Darlow, Hilbert transform techniques for measurement of transient gear speeds, Mechanical Systems and Signal Processing 2 (2) (1988) 187-194.

[18] F. Combet, L. Gelman, An automated methodology for performing time synchronous averaging of a gearbox signal without speed sensor, Mechanical Systems and Signal Processing 21 (6) (2007) 2590-2606.

[19] F. Combet, R. Zimroz, A new method for the estimation of the instantaneous speed relative fluctuation in a vibration signal based on the short time scale transform, Mechanical Systems and Signal Processing 23 (4) (2009) 1382-1397.

[20] J. Klapper, J.T. Frankle, Phase-Locked and Frequency-Feedback Systems, Academic Press, New York, 1972.

[21] Z. Bin, Z. Dong-lai, Research on synchronous sampling clock jitter of power system, Proceedings of the 2nd International Conference on Information Engineering and Computer Science (ICIECS), 25 Dec-26 Dec 2010, Wuhan, China.

[22] R. Potter, M. Gribler, GRIBLER, Computed order tracking obsoletes older methods, Proceedings of the SAE Noise and Vibration Conference, 1989, pp. 6367.

[23] W. Potter, Tracking and resampling method and apparatus for monitoring the performanceof rotating machines, United States Patent 4,912,661, 1990.

[24] R. Potter, A new order tracking method for rotating machinery, Sound and Vibration 24 (1990) 30-34. 
[25] D. Rémond, J. Antoni, R.B. Randall, Editorial for the special issue on Instantaneous Angular Speed (IAS) processing and angular applications, Mechanical Systems and Signal Processing 44 (1-2) (2014) 1-4.

[26] S. Braun, The synchronous (time domain) average revisited, Mechanical Systems and Signal Processing 25 (4) (2011) $1087-1102$.

[27] S. Braun, Mechanical Signature Analysis, Academic Press, 1986.

[28] D. Abboud, S. Baudin, J. Antoni, D. Remond, M. Eltabach, O. Sauvage, The spectral correlation analysis of cyclo-non-stationary signals, Mechanical Systems and Signal Processing (2015).

[29] M. Rosenblatt, Remarks on some nonparametric estimates of a density function, Annals of Mathematical Statistics 27 (3) (1956) 832.

[30] E. Parzen, On estimation of a probability density function and mode, Annals of Mathematical Statistics 33 (3) (1962) 1065.

[31] Birge, L., Rozenholc, Y. How many bins should be put in a regular histogram, Prepublication no 721, Laboratoire de Probabilites et Modeles Aleatoires, CNRS-UMR 7599, Universite Paris VI \& VII, 2002.

[32] P. Hall, E. Hannan, On stochastic complexity and nonparametric density estimation, Biometrika 75 (4) (1988) $705-714$.

[33] H. André, F. Girardin, A. Bourdon, J. Antoni, D. Rémond, Precision of the IAS monitoring system based on the elapsed time method in the spectral domain, Mechanical Systems and Signal Processing 44 (1-2) (2014) 14-30.

[34] Q. Leclère, L. Pruvost, E. Parizet, Angular and temporal determinism of rotating machine signals: the diesel engine case, Mechanical Systems and Signal Processing 24 (7) (2010) 2012-2020.

[35] R.B. Randall, J. Antoni, Rolling element bearing diagnostics-a tutorial, Mechanical Systems and Signal Processing 25 (2) (2011) $485-520$. 